\title{
Philosophiques
}

\section{La théorie générale des modes verbaux dans les grammaires philosophiques de l'époque classique}

\section{André Leclerc}

Volume 15, numéro 2, automne 1988

URI : https://id.erudit.org/iderudit/027051ar

DOI : https://doi.org/10.7202/027051ar

Aller au sommaire du numéro

\section{Éditeur(s)}

Société de philosophie du Québec

\section{ISSN}

0316-2923 (imprimé)

1492-1391 (numérique)

Découvrir la revue

\section{Citer cet article}

Leclerc, A. (1988). La théorie générale des modes verbaux dans les grammaires philosophiques de l'époque classique. Philosophiques, 15(2), 331-387. https://doi.org/10.7202/027051ar

\section{Résumé de l'article}

C'est dans leur théorie générale des modes verbaux que les grammairiens philosophes classiques (circa 1660-1800) ont essayé de rendre compte de ce que nous appelons aujourd'hui les " aspects illocutoires de la signification ", et de formuler les principes d'une sémantique des énoncés non déclaratifs. Je voudrais montrer, dans cette étude, ce qui fait l'originalité de la position adoptée par les grammairiens philosophes dans le cadre de leur théorie " idéationnelle » du langage : tout en reconnaissant la nécessité de recourir à des critères formels pour identifier un mode particulier dans une langue particulière, ils estimaient que les peuples auraient pu, s'ils l'avaient jugé utile, marquer et distinguer formellement toute la variété des types d'illocution par des flexions verbales caractéristiques. Je distingue deux approches dans la théorie générale des modes verbaux de la Grammaire Générale classique : l'une est réductionniste, parce qu'elle réduit toutes nos énonciations à des expressions de jugements ; l'autre conçoit les modes comme des marqueurs d'actes de pensée. La première rappelle les tentatives de ceux qui, comme D. Lewis et D. Davidson, cherchent à interpréter les énoncés non déclaratifs en leur assignant des conditions de vérité ; la seconde s'apparente plutôt à la Théorie des Actes de Discours ou à l'approche proposée par E. Stenius. La première partie de cette étude présente, en s'inspirant de la méthodologie de I. Lakatos, la Grammaire Générale classique comme un programme de recherche scientifique. La seconde partie présente les deux approches mentionnées et examine leurs dévelop- pements respectifs.
Tous droits réservés @ Société de philosophie du Québec, 1988
Ce document est protégé par la loi sur le droit d'auteur. L'utilisation des services d'Érudit (y compris la reproduction) est assujettie à sa politique d'utilisation que vous pouvez consulter en ligne.

https://apropos.erudit.org/fr/usagers/politique-dutilisation/ 


\title{
LA THÉORIE GÉNÉRALE DES MODES VERBAUX DANS LES GRAMMAIRES PHILOSOPHIQUES DE L'ÉPOQUE CLASSIQUE ${ }^{1}$
}

\author{
par André Leclerc
}

RÉSUMÉ. C'est dans leur théorie générale des modes verbaux que les grammairiens philosophes classiques (circa 1660-1800) ont essayé de rendre compte de ce que nous appelons aujourd'hui les "aspects illocutoires de la significarion", et de formuler les principes d'une sémantique des énoncés non déclaratifs. Je voudrais montrer, dans cette étude, ce qui fait l'originalité de la position adoptée par les grammairiens philosophes dans le cadre de leur théorie " idéationnelle " du langage: tout en reconnaissant la nécessité de recourir à des critères formels pour identifier un mode particulier dans une langue particulière, ils estimaient que les peuples auraient pu, s'ils l'avaient jugé utile, marquer et distinguer formellement toute la variété des types d'illocution par des flexions verbales caractéristiques. Je distingue deux approches dans la théorie générale des modes verbaux de la Grammaire Générale classique : l'une est réductionniste, parce qu'elle réduit toutes nos énonciations à des expressions de jugements; l'autre conçoit les modes comme des marqueurs d'actes de pensée. La première rappelle les tentatives de ceux qui, comme $\mathrm{D}$. Lewis et D. Davidson, cherchent à interpréter les énoncés non déclaratifs en leur assignant des conditions de vérité ; la seconde s'apparente plutôt à la Théorie des Actes de Discours ou à l'approche proposée par E. Stenius. La première partie de cette étude présente, en s'inspirant de la méthodologie de I. Lakatos, la Grammaire Générale classique

1. Ce texte est une version revue et augmentée d'une communication présentée au Congrès de l'A.C.F.A.S. tenu en mai 1986 à l'Université de Montréal. Je remercie vivement MM. S. Auroux, N. Kaufman, J. Naud, C. Panaccio, C. Savary et $D$. Vanderveken pour leurs commentaires et encouragements. Comme le veut la tradition dans ces circonstances : rien de ce qui est écrit dans cet article ne pourra être retenu contre eux ! Cette recherche a été rendue possible grâce à l'aide financière du F.C.A.R. et du C.R.S.H.C. Je tiens ici à leur exprimer toute ma gratitude. 
comme un programme de recherche scientifique. La seconde partie présente les deux approches mentionnées et examine leurs développements respectifs.

ABSTRACT. The Grammairiens philosophes of the classical period (circa 1660-1800) tried to accommodate, in their general theory of the moods of verbs, what we call today the "illocutionary aspects of meaning ", and to set the principles of a semantics for non-declarative sentences. I would like to show in this study in what consists the distinctive character of the approach proposed by the grammairiens philosopbes in the framework of their «ideational» theory of language: they accept the necessity of formal criteria to identify a particular mood in a particular language, but they also held up the following position: the nations could have, if they had found it useful, marked and distinguished formally all the variety of illocution types by the use of characteristic verbal inflexions. I distinguish two approaches in the general theory of the verbal moods of classical Universal Grammar : one of them is reductionist, because it analyses all kinds of illocution as an expression of a judgment ; the other one conceives the moods as markers of the acts of the mind. The first one recalls the approach of those who, like $\mathrm{D}$. Lew is and $\mathrm{D}$. Davidson, try to interprete non-declarative sentences by assigning them truthconditions; and the second one bears more resemblances with the Theory of Speech Acts, or with the position defended by E. Stenius. The first part of this study exposes the framework of the classical Universal Grammar as a scientific research programme, following the methodology of I. Lakatos. The second part deals with the two approaches just mentioned and examine their respective developments.

\section{Introduction}

Les modes verbaux sont familiers (ou devraient l'être...) à toute personne ayant fréquenté la petite école ${ }^{2}$. Le français, par exemple, en compte six: quatre modes personnels (indicatif, impératif, conditionnel et subjonctif), et deux modes impersonnels (infinitif et participe). Mais le nombre et la nature des modes

2. Quintillien écrivait, dans son Institution oratoire (Tome 1, Paris, éd. Garnier Frères, 1954) : "Quant aux verbes, maintenant, est-il homme assez peu cultivé pour ignorer qu'ils ont des voix, des modes, des personnes et des nombres? C'est à peu près ce qu'on apprend dans les écoles primaires; ce sont des connaissances élémentaires. Mais il y a des phénomènes qui étonneront, parce que la flexion en est équivoque. " (P. 63) 
peuvent varier d'une langue à une autre. Ainsi, le latin et le grec n'ont pas de conditionnel, le grec ancien a un optatif, l'allemand a deux subjonctifs, le chinois n'a pas, à proprement parler, de flexion verbale pour les modes, et des grammairiens discutaient encore récemment sur la reconnaissance d'un soi-disant mode «présomptif» en roumain ${ }^{3}$.

Toutes les grammaires générales de l'époque classique, de Port-Royal (la Grammaire générale et raisonnée est de 1660) à Destutt de Tracy (sa Grammaire paraît en 1803), contiennent une théorie générale des modes verbaux. C'est dans le cadre de cette théorie que les grammairiens philosophes ont abordé les questions relatives à la syntaxe (théorie de la «construction»), à la sémantique et à la pragmatique des énoncés déclaratifs et non déclaratifs. En effet, la plupart des grammairiens philosophes n'hésitaient pas à parler de modes verbaux interrogatif, dubitatif, déprécatif ou concessif, même si aucune flexion verbale ne leur correspond dans les langues qu'ils connaissaient. Les linguistes contemporains insistent (par exemple Zaefferer ${ }^{4}$, 1984a) sur la distinction que l'on se doit de faire entre les modes verbaux (indicatif, impératif, subjonctif, conditionnel, optatif, infinitif, participe, etc.) et les modes d'énoncé (déclaratif, jussif ou impératif, interrogatif, dubitatif, optatif, promissif, exclamatif, etc.), même si les deux catégories se recoupent en plusieurs points (indicatif-déclaratif, impératif-jussif, «optatif» désignant aussi bien un mode verbal qu'un mode d'énoncé). Les modes verbaux relèvent de la morpho-syntaxe, tandis que les modes d'énoncé (sentence moods, Satzmodi) sont habituellement pris en charge par l'une ou l'autre des trois disciplines de la célèbre tripartition de Morris. Mais si la distinction mode verbal/mode d'énoncé doit être strictement maintenue, comme le veut $Z$ aefferer, le développement historique de ces deux

3. Cf., Vladimir FloRA, «Existe-t-il un mode présomptif en roumain? 》, in Langage et psychomécanique du langage (pour Roch Valin), éd. par A. Joly et W.H. Hirtle, les Presses de l'Université de Lille, 1980.

4. Dietmar Zaefferer et Günther Grewendorf, "Theorien der Satzmodi», art. du manuel Semantik, éd. par D. Wunderlich et A. von Stechow; manuscrit, juin 1984; "Von dem Satzmodi streng unterscheiden sind die Modi des Verbs (Indikativ, Konjunktiv, Imperativ, etc.), obwohl oder gerade weil zum Teil, z.B. beim Imperativ (Verb- und Satzmodus) enge Zusammenhange bestehen ». 
notions montre à quel point elles sont intimement liées. D'après Nuchelmans (1973), la théorie des modes verbaux des premiers grammairiens grecs (Denys de Thrace, Apollonios Dyscole) s'inspirerait directement, du moins en ce qui a trait aux modes personnels, des classifications des modes d'énoncé (ou " genres de discours ») avancées par les péripatéticiens et les stö̈ciens 5 . D'ailleurs, les recoupements mentionnés plus haut entre les deux catégories furent probablement la source des nombreuses confusions entre les modes verbaux et les modes d'énoncé survenues au cours de l'Antiquité et du Moyen âge, chez des auteurs aussi influents que Martianus Capella, Boèce, Guillaume de Sherwood et Pierre d'Espagne ${ }^{6}$.

Je voudrais, dans cette étude, montrer l'originalité du point de vue adopté par les grammairiens philosophes dans leur théorie générale des modes verbaux : tout en reconnaissant la nécessité de recourir à des critères formels (morphologiques) pour identifier un mode verbal particulier dans une langue particulière, les grammairiens philosophes soutiennent par ailleurs qu'en théorie, les peuples auraient pu, s'ils l'avaient jugé utile, marquer et distinguer formellement toute la variété des modes d'énoncé par des flexions verbales caractéristiques. Les modes verbaux et les modes d'énoncé ne sont, pour l'essentiel, que deux moyens, différents mais équivalents, d'atteindre les mêmes fins : exprimer conventionnellement différents actes de pensée (ou différentes opérations de l'esprit), tels l'affirmation, l'interrogation, le commandement, le désir (ou le souhait), le doute, etc. Les grammairiens philosophes affirment souvent qu'il y a, pour ainsi dire, une bijection entre les modes verbaux possibles et les modes d'énoncé possibles. C'est pourquoi, dans la Grammaire Générale classique, les modes d'énoncé (ou types d'illocution) sont presque toujours traités au chapitre des modes verbaux. Par ailleurs, nos grammairiens philosophes ont tendance à assimiler les modes impersonnels soit aux substantifs (infinitif), soit aux adjectifs (participe). En assimilant de la sorte les modes d'énoncé aux modes verbaux personnels, et les modes

5. Cf. G. Nuchelmans, Theories of propositions, Ancient and Medieval Conceptions of the Bearers of Truth and Falsety, Amsterdam, North-Holland, 1973, p. 101. 
impersonnels aux substantifs et adjectifs, les grammairiens philosophes semblent avoir simplement placé sous la catégorie traditionnelle du mode verbal les questions relatives aux modes d'énoncé, déclaratifs et non déclaratifs. Cette double tendance, qui consiste à inclure les modes d'énoncé dans la catégorie des modes verbaux et à en exclure les modes impersonnels, délimite un objet qui nous paraît fort semblable à celui de la Théorie des Actes de Discours et des théories actuelles de l'énonciation. La Théorie des Actes de Discours et la théorie générale des modes verbaux de la Grammaire Générale visent au fond la même cible : rendre compte des aspects illocutoires de la signification, ces aspects de la signification qui déterminent si l'énonciation littérale d'une phrase doit compter comme assertion, témoignage, prédiction, question, ordre, prière, requête, promesse, exclamation, etc. Même si les grammairiens philosophes ne disposaient pas des concepts d'acte et de force illocutoires, nous verrons que le concept d'acte de pensée, et celui d'opération sociale de l'esprit développé tardivement par les common sense philosophers écossais, remplissent à peu près les mêmes fonctions dans la Grammaire Générale. Mon hypothèse de travail pourrait donc se formuler ainsi : le mode du verbe constitue, dans la Grammaire Générale classique, le principal marqueur de force illocutoire. Plus précisément, le mode du verbe indique le but illocutoire d'une énonciation, car des énonciations marquées par le même mode peuvent avoir des forces illocutoires distinctes (e.g. : une prédiction et un témoignage ont le même but illocutoire et le même mode verbal : l'indicatif; de même, l'impératif peut servir à exprimer un ordre, un commandement, une prière ou une concession). Plusieurs raisons militent en faveur de cette hypothèse. Nous y reviendrons à la toute fin. Mais d'abord, je crois utile de présenter brièvement le «cadre théorique» des grammairiens philosophes, et leurs principales stratégies d'explication de «ce qui est commun à toutes les langues» (Port-Royal). Ensuite, j'examinerai plus à fond la théorie générale des modes verbaux, en distinguant les différentes approches qui ont été proposées par les grammairiens philosophes, et les principales étapes qui ont marqué le développement de cette théorie pendant les cent cinquante ans au cours desquels s'épanouit le mouvement de la Grammaire Générale en France et en Grande-Bretagne. 


\section{LA GRAMMAIRE GÉNÉRALE CLASSIQUE EN TANT QUE PROGRAMME DE RECHERCHE SCIENTIFIQUE}

La description du mouvement de la Grammaire Générale que jentreprends ici s'inspire largement des principes de la métbodologie des programmes de recherche scientifique proposée par Imre Lakatos pour l'historiographie des sciences ${ }^{7}$.

Un programme de recherche scientifique est un ensemble de règles métbodologiques prescrivant les voies de recherche à éviter (beuristique négative) et celles qui sont à parcourir (beuristique positive). L'heuristique négative détermine le noyau dur (bard core), un ensemble de lois, de principes ou de conceptions théoriques qui sont déclarés inattaquables par une décision méthodologique des chercheurs qui s'engagent dans un programme de recherche particulier. Le noyau dur se trouve ainsi soustrait aux procédures de falsification. L'heuristique positive, elle, fixe un programme destiné à construire une ceinture de protection (protective belt) autour du noyau dur, un ensemble de théories ou d'hypothèses auxiliaires qui sont, elles, susceptibles d'être falsifiées, et qui peuvent être ajustées, modifiées, ou même abandonnées au profit d'autres hypothèses ou théories sans que le noyau dur ait à en subir les contrecoups. Le lien entre le noyau dur et les hypothèses auxiliaires n'est donc pas déductif; les hypothèses auxiliaires doivent «s'inspirer » du noyau dur ou du moins doivent lui être compatibles. Un programme de recherche scientifique ne s'identifie pas à une théorie particulière, mais plutôt à une série de théories ayant en commun le même noyau dur. Dans les pages qui suivent, j'insisterai davantage sur l'heuristique négative (le noyau dur) de la Grammaire Générale que sur son heuristique positive, parce qu'une description complète de la ceinture de protection dépasserait largement le cadre de cet article ${ }^{8}$.

6. Ibid., p. 166 et passim.

7. Cf. I. LAKATOS, «The Methodology of Scientific Research Programmes» in Philosophical Papers, vol. 1, éd., par J. Worrall er G. Currie, Cambridge : U.P., 1978, pp. 8-101.

8. Le lecteur désireux d'en savoir plus sur le mouvement de la Grammaire Générale consultera avec profit les ouvrages suivants: $H$. A ARSLEFF, The Study of Language in England, 1780-1860, Princeton, Princeton U.Press., 1967; A. AARSLEFF, From Locke to Saussure, Minneapolis, U. of Minnesota Press, 1982; S. Auroux, L'Encyclopédie. "Grammaire" et "langue» au XVIII siècle, Paris, Mame, 1973; S. AUROUX, La 
La Grammaire Générale classique se présente comme une science, que les grammairiens philosophes opposaient volontiers à l'art grammatical qui, lui, se limite à l'étude des grammaires particulières. On trouve cependant aussi des grammaires particulières (françaises, par exemple), écrites par des grammairiens philosophes en respectant les principes de la Grammaire Générale - c'est le cas de la Grammaire françoise sur un plan nouveau (1709) de Claude Buffier, des Véritables principes de la langue françoise (1747) de Gabriel Girard, et de la seconde partie de la Grammaire (1775) de Condillac. L'un des plus illustres représentants du mouvement de la Grammaire Générale, l'encyclopédiste Beauzée, disait de la Grammaire Générale qu’elle est « la science raisonnée des principes immuables et généraux du Langage prononcé ou écrit, dans quelque langue que ce soit»; qu'elle est "une science, parce qu'elle n'a pour objet que la spéculation raisonnée des principes immuables et généraux du Langage »; et que la «science grammaticale est antérieure à toutes les langues, parce que ses principes ne supposent que la possibilité des langues, qu'ils sont les mêmes que ceux qui dirigent la raison humaine dans ses opérations intellectuelles; en un mot, qu'ils sont d'une vérité éternelle »(Grammaire générale, 1767, pp. V-VI). D'autres grammairiens philosophes, au tempérament moins rationaliste que Beauzée, préfèrent parler d' «observations » qui conviennent à toutes les langues. Buffier (1709), par exemple, dit clairement que

Sémiotique des Encyclopédistes, Paris, Payot, 1979; J.C. CheVALIER, Histoire de la syntaxe. Naissance de la notion de complément dans la grammaire française (1530-1750), Genève, Droz, 1968; N. ChOMSKy, La Linguistique cartésienne, Paris, Seuil, 1969; M. Dominicy, La Naissance de la grammaire moderne, Bruxelles, Pierre Mardaga, 1984 ; R. DONZÉ, La Grammaire générale et raisonnée de Port-Royal, Berne, Francke, 1967; A. Joly et J. Stefanini (éd.), La Grammaire générale. Des Modistes aux Idéologues, Lille, Presses Universitaires de Lille, 1977 ; I. MICHAEL, English Grammatical Categories and the Tradition to 1800, Cambridge, C.U.P., 1970; G. NuCHELMans, Judgment and Proposition. From Descartes to Kant, Amsterdam, North-Holland, 1983 ; G.A. PADlEY, Grammatical Theory in Western Europe, 1500-1700, Cambridge, C.U.P., 1985 ; H. PARRET, History of Linguistic Thought and Contemporary Linguistics, Berlin-New York, de Gruyter, 1976; J.C. PARIENTE, L'Analyse du langage à PortRoyal. Six études logico-grammaticales, Paris, Ed. du Minuit, 1985; U. Ricken, Grammaire et philosophie au siècle des Lumières. Controverse sur l'ordre narurel et la clarté du français, Villeneuve-d'Ascq, Université de Lille III, 1978; G. SAHLIN, César Chesneau Du Marsais et son rôle dans l'évolution de la grammaire générale, Paris, P.U.F., 1928 ; J. SGard (éd.), Condillac et les problèmes du langage, Genève et Paris, Slatkine, 1982. 
la grammaire particulière d'une langue n'est pas une discipline normative prescrivant «le bon usage »; sa tâche est de décrire et d'expliquer les usages effectifs d'une communauté de sujets parlants.

La Grammaire Générale classique cherche à déterminer quels sont les types ou catégories d'expressions (partes orationis) qui sont nécessaires et/ou suffisants à l'expression complète et adéquate de la pensée dans toutes les langues bumaines. Sa tâche est de rendre compte « de ce qui est commun à toutes les langues » (PortRoyal). La Grammaire Générale se caractérise donc par un souci d'adéquation explicative. Elle est, écrit M. Auroux, une théorie «contenant les principes de la construction des énoncés dans toutes les langues » 9. La Grammaire Générale peut aussi être décrite comme l'étude de deux types de contraintes: les unes concernent les conditions de la représentation de la pensée dans la langue ; les autres, les conditions de la communication de la pensée dans le discours. La Grammaire Générale est ainsi l'étude des contraintes que doit satisfaire tout système de signes pour représenter complètement la pensée, et pour la communiquer efficacement, c'est-à-dire clairement et sans trop d'embarras. L'idéologue Destutt de Tracy (Grammaire, 1803, p. 67), après avoir examiné les parties dont la proposition "est nécessairement composée», se propose « d'examiner les différentes sortes de mots dont on se sert dans nos langues perfectionnées, pour rendre l'expression de la pensée plus complète et plus facile». (Je souligne).

Une grammaire générale comprend une partie portant sur «les sons et les lettres», c'est-à-dire la «face matérielle»du langage. Cette partie, qui occupe habituellement moins du quart des grammaires générales, correspond grosso modo à ce que nous appelons aujourd'hui «phonétique » ou «phonologie ». On y trouve une description de l'appareil phonatoire, des principaux organes qui contribuent à la production des voyelles et des consonnes, des rapports entre les lettres, les sons et les idées, et diverses règles touchant la prosodie. Les autres parties examinent la « face spirituelle » du langage, que se partagent la syntaxe, la sémantique et la

9. S. Auroux, «Le temps verbal dans la Grammaire Générale », chap. 1 de Innovation et système, manuscrit. 
pragmatique. La syntaxe est le niveau le moins développé ; les grammairiens philosophes, qui héritaient de la vieille distinction syntaxe de convenance/syntaxe de régime, ont néanmoins fait évoluer les choses en travaillant les rapports de détermination et d'explication (pour les propositions relatives), et en délimitant des niveaux de « construction » en syntaxe, comme le fait Dumarsais (constructions « naturelle», «figurée » et « usuelle»). La pragmatique, et surtout la sémantique, retiennent davantage l'attention des grammairiens philosophes. La théorie des parties du discours (partes orationis) constitue le cour des grammaires générales. Les noms (propres et appellatifs, et leurs cas), pronoms, adjectifs, verbes, adverbes, participes, articles, prépositions, conjonctions et interjections y sont traités séparément, mais jamais indépendamment de leur contribution à l'expression conventionnelle d'une pensée complète. Le point de vue de la Grammaire Générale est celui de la fonction ; c'est le rôle ou la contribution sémantique d'une expression qui détermine son appartenance à telle ou telle classe de mots. Chez la plupart des grammairiens philosophes, les critères purement syntaxiques ou morphologiques se font rares.

Le noyau dur de la Grammaire Générale classique se réduit à mon avis aux quelques principes suivants:

1) Le langage est l'expression (ou l'analyse) de la pensée (définition);

2) La principale fonction du langage est la communication des pensées;

3) La pensée est la même partout et pour tous (postulat de l'universalité de la pensée) ;

4) L'usage de la parole est une activité rationnelle orientée vers une fin (principe de rationalité appliqué à l'usage du langage);

5) Le principe d'analogie : les langues se forment et évoluent en respectant certains modèles stables (comme les paradigmes de déclinaison et de conjugaison), sans quoi elles deviennent irrégulières et difficiles à apprendre;

6) Il $y$ a des universaux substantiels: pour exprimer (ou analyser) complètement la pensée, toutes les langues bumaines ont besoin sensiblement des mêmes catégories. 
Examinons maintenant ces principes.

1) La définition du langage comme expression (conventionnelle) de la pensée est, pour l'essentiel, aristotélicienne. Les mots sont les signes des idées (des «états de l'âme», disait Aristote dans le Traité de l'interprétation). Le même principe se retrouve chez Augustin (par exemple, Les Confessions, Livre 10, chapitre XII). Il est à la base de la théorie des signes de logiciens et grammairiens de Port-Royal, et peu de philosophes l'ont autant développé et discuté que Locke dans le troisième Livre de son Essay Concerning Human Understanding. La Grammaire Générale classique est une théorie «idéationnelle» du langage ${ }^{10}$; sa principale stratégie d'explication des faits de langue consiste à les rapporter à des opérations de l'esprit, principalement le jugement, mais aussi à la conception (qui produit les idées ou concepts), et à tous les "mouvements de l'âme", selon l'expression des Messieurs de Port-Royal, comme le désir, l'interrogation, le commandement, la prière, etc. Nous reviendrons plus tard sur la logique et la psychologie sur lesquelles s'appuie la Grammaire Générale. Notons simplement que la sémiologie des Lumières s'élève sur une structure ternaire: un signe est quelque chose (un mot écrit ou prononcé par exemple) qui tient lieu d'une idée dans l'esprit du locuteur, laquelle représente une chose dans le monde. Le monde se divise en choses, la pensée en idées, et les discours en mots. Les mots sont les signes des idées qui sont des représentations des choses.

2) Plusieurs philosophes classiques (Locke, Leibniz, Berkeley, Condillac) admettent volontiers que le langage a d'autres fins que la communication des pensées (par exemple, il sert de support à la mémoire pour les "pensées abstraites ", à raisonner à part soi et en silence, et il «donne de l'exercice » aux facultés supérieures de l'âme). Mais tous admettent (sauf Berkeley) que sa principale fonction est la communication des pensées, et le point de vue exprimé par Leibniz est bien représentatif de la période examinée

10. J'emprunte ce terme à W. ALSTON, The Pbilosophy of Language, Englewood cliffs, Prentice-Hall, 1964, pp. 11 et suiv. Le principe des théories idéationnelles du langage est clairement formulé dans la Grammaire générale et raisonnée : "La connoissance de ce qui se passe dans notre esprit est nécessaire pour comprendre les fondemens de la Grammaire». (P. 26) 
ici : «sans le désir de nous faire entendre nous n'aurions jamais formé de langage » (Nouveaux essais..., Livre III, p. 236). Dans l'anthropologie classique, Dieu a créé l'homme pour la vie en société, avec une âme rationnelle et les organes de la parole. Est-ce le langage qui rend la société possible ou l'inverse? La question fut débattue au XVIII ${ }^{\mathrm{e}}$ siècle, mais à tous le langage apparaît comme le ciment de la vie sociale.

3) Le postulat de l'universalité de la pensée est évidemment très important pour la Grammaire Générale. Le langage pourrait être l'expression de la pensée sans que la pensée soit la même partout et pour tous. Si c'était le cas, la Grammaire Générale serait sans fondement. Par "pensée », il faut entendre ici avant tout la pensée conceptuelle, par opposition à l'imagination et à la sensation. À la suite de Descartes (voir les premiers paragraphes de la $\sigma^{e}$ Méditation), les Messieurs distinguent clairement, au début de leur Logique ou l'art de penser (1662), l'imagination d'un polygone à 1996 côtés, qui ne produit qu'une image mentale confuse, de la conception du même polygone qui, elle, produit une idée claire et distincte, une idée qui est bien la même pour tous les géomètres de toutes les époques. Ainsi conçue, la pensée devait être la même partout et pour tous. Les hommes ont partout les mêmes besoins fondamentaux, et partout les idées se forment sur les mêmes principes. Ainsi, les mots qui expriment en différentes langues des idées complexes doivent se correspondre assez étroitement. David Hume exprime clairement cette conception :

Dans différentes langues, même dans celles entre lesquelles nous ne pouvons soupçonner la moindre connexion ou communication, on trouve que les mots significatifs des idées les plus complexes se correspondent étroitement : preuve certaine que les idées simples, comprises dans les idées complexes, sont liées par un principe universel d'influence égale sur tous les hommes. ${ }^{11}$

11. David Hume, Enquête sur l'entendement humain, Paris, Aubier, éd. Montaigne, 1947, p. 59. Les principes universels dont parle Hume sont les principes d'association selon la ressemblance, la contiguité et la causalité. Ces principes, renforcés par l'effet de l'accoutumance, assurent la liaison des idées ; celle-ci n'est donc pas le fait d'une Raison éternelle et transhistorique. Mais Hume (ibid., pp. 60-61) présente néanmoins une conception de la raison pratique qui est tout à fait semblable à celle que j'essaierai de reconstruire plus loin : «Comme l'homme est un ètre raisonnable et qu'il est continuellement à la recherche du bonheur, qu'il espère atteindre par la satisfaction d'une 
Le seul philosophe classique qui ait osé mettre en doute le principe de l'universalité de la pensée est Maupertuis, dans ses Réflexions philosophiques sur l'origine des langues et la signification des mots (1748). Cet opuscule, qui paraît la même année que l'Enquête surl'entendement bumain de Hume, contient en effet ce qui peut passer pour la première formulation du principe de relativité linguistique:

... on trouve des Langues, sur-tout chez les peuples fort éloignés, qui semblent avoir été formées sur des plans d'idées si différents des nôtres, qu'on ne peut presque pas traduire dans nos Langues ce qui a été une fois exprimé dans celles-là. ${ }^{12}$

Les «plans d'idées différents» de Maupertuis furent immédiatement condamnés par les philosophes français qui avaient eu la chance de lire l'opuscule dont à peine une douzaine d'exemplaires circulaient. La réaction de Turgot (un empiriste) rejoint les idées exprimées par Hume dans le passage cité plus haut:

Les plans d'idées différens sont de l'invention de Maupertuis. Tous les peuples ont les mêmes sens, et sur les sens se forment les idées : aussi nous voyons les fables même de tous les peuples se ressembler beaucoup. ${ }^{13}$

La réaction de Condillac aux "plans d'idées différents» de Maupertuis ira dans le même sens. Dans une lettre adressée à Maupertuis (datée du 25 juin 1752), il écrit :

Il y auroit de la différence entre la philosophie de deux peuples qui n'auroient eu aucun commerce ensemble, et la différence des langages pourroit y contribuer : je doute cependant que cette différence fût aussi

passion ou d'une affection, il agit, parle ou pense rarement sans but ni intention. Il a toujours quelque objet en vue ; et, quelle que soit parfois l'impropriété des moyens qu'il choisit pour atteindre sa fin, il ne perd jamais celle-ci de vue et il ne laissera même pas se perdre ses pensées et ses réflexions quand il n'espère pas en obtenir quelque satisfaction".

12. On trouve le texte de Maupertuis dans Charles PORSET (éd.) Varia linguistica, Bordeaux, Éditions Ducros, 1970; la citation est tirée de la page 27. J'ai examiné brièvement la controverse soulevée par cette affirmation de Maupertuis dans «Le problème de la traduction au siècle des Lumières: obstacles pratiques et limites théoriques », in Traduction, Terminologie, Rédaction, vol. 1, numéro 1, pp. 41-62.

13. A.-R. TURGot, « Remarques critiques sur les Réflexions philosophiques de Maupertuis sur l'origine des Langues et la signification des mots », in Varia linguistica de C. Porset (éd.), p. 26. 
considérable que vous paroissez le supposer, les hommes ayant partout les mêmes sens et des besoins semblables: je crois que sans se communiquer, ils seroient sûrement conduits à faire les mêmes abstractions et les mêmes raisonnements. ${ }^{14}$

Un demi-siècle plus tard, Maine de Biran rejoindra le groupe des critiques de Maupertuis ${ }^{15}$. De toute évidence, le postulat de l'universalité de la pensée n'est pas de ceux que l'on remet en question lorsquion est grammairien philosophe. Plusieurs « hyporhèses auxiliaires » de la Grammaire Générale (théorie de la traduction, théorie des idées accessoires, théories de l'inversion et de l'origine des langues) visent en partie à mettre ce postulat à l'abri de contre-exemples par trop évidents.

Il convient je crois de toucher ici quelques mots à propos de la logique et de la théorie de l'esprit ( « théorie des idées », « psychologie rationnelle", ou encore "Idéologie ») de l'âge classique. Car les deux disciplines sur lesquelles se fonde la Grammaire Générale sont très liées du fait qu'elles s'occupent toutes les deux, mais chacune à leur manière, de la "pensée". La logique s'intéresse avant tout à certaines opérations de l'esprit (concevoir, juger, raisonner, ordonner), dans la mesure ou ces opérations, conduites sous la droite raison, nous rendent capables d'atteindre la vérité dans les sciences. La pensée qui importe aux yeux des grammairiens philosophes est celle que produisent les deux premières opérations : la conception, qui donne les concepts ou idées, et le jugement, qui produit une pensée susceptible d'être vraie ou fausse. Les idées deviennent les significations des mots lorsqu'elles sont associées aux mots par convention. Les jugements, eux, s'expriment par des propositions déclaratives, composées d'un sujet, d'un prédicat (ou attribut), et le plus souvent d'une copule ${ }^{16}$. Il y a une nette primauté du jugement chez les philosophes classiques, puisqu'on ne parle guère pour dire ce que l'on conçoit, mais plutôt presque

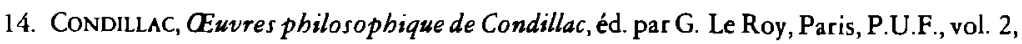
1948 , in Correspondance, p. 537.

15. Maine De Biran, "Note sur les Réflexions de Maupertuis et Turgot au sujet de l'origine des langues ", in R. Grimsley (éd.), Sur l'origine du langage, Genève, Droz, 1971.

16. On trouve cependant plusieurs analyses bipartites de la proposition au XVIIIe siècle ; cf. G. Nuchelmans, Judgment and Proposition. From Descartes to Kant, Amsterdam, North-Holland, 1983 ; en particulier les chapitres 5 et 9. 
toujours pour dire ce que l'on juge des objets conçus. De plus, raisonner, au fond, c'est simplement former un nouveau jugement à partir de jugements déjà donnés. Parce que les jugements s'expriment dans le discours par des propositions, la «proposition considérée grammaticalement » (selon l'expression de Dumarsais) constitue l'unité maximale d'analyse dans la Grammaire Générale. L'unité de la proposition (considérée grammaticalement) est la représentation du jugement. L'analyse de la proposition dans la Grammaire Générale sera entièrement empruntée à la logique, avec toutefois des modifications importantes : en devenant grammaticale, la proposition n'est plus seulement porteuse des valeurs de vérité, elle se fait aussi principale, incidente ou subordonnée ${ }^{17}$. La théorie des propositions que la Grammaire Générale emprunte à la logique ne reconnaît pas l'existence des relations et cherche à ramener toutes les propositions à la forme générale Sujet-CopulePrédicat. Les propositions peuvent varier selon la qualité (affirmative ou négative), ou selon la quantité (universelle, particulière ou singulière). On trouve cependant des analyses bipartites de la proposition (en Sujet-Prédicat) chez Buffier, Dumarsais, Beauzée et Destutt de Tracy; la copule est alors une partie essentielle de l'attribut.

Si la logique classique est essentiellement aristotélicienne, la psychologie classique a plutôt des allures cartésiennes. La plupart des auteurs que nous considérons ici acceptent en effet la distinction cartésienne entre l'âme et le corps ; c'est le cas, par exemple, d'empiristes avoués comme Dumarsais ${ }^{18}$ et Condillac ${ }^{19}$, qui suivent par ailleurs Locke dans son rejet des idées innées et l'importance qu'il attache aux sens et à l'expérience. C'est davantage la genèse des idées et connaissances que la nature de l'esprit qui est en cause dans la querelle opposant les sensualistes aux rationalistes. La théorie classique de l'esprit se donne principalement pour tâche de déterminer la nature de l'esprit, de ses facultés et de ses opérations, et d'étudier la genèse de nos idées et connaissances. L'âme des classiques diffère grandement de celle d'Aristote. Sous l'influence

17. Cf. S. AurouX, "Le temps verbal dans la Grammaire Générale », manuscrit, op. cit.

18. DuMARSAIS reprend la distinction cartésienne dès le premier article de sa Logique. Cf. Logique et Principes de Grammaire, paru à titre posthume en 1769.

19. Condillac, Essai sur l'origine des connoissances bumaines, in Cuvres philosophiques de Condillac, vol. 1, 1947. Section première, chapitre premier. 
de Descartes, l'âme se voit retirer toute fonction vitale. L'explication de ces fonctions vitales (respiration, digestion, circulation sanguine, etc.) est maintenant l'affaire de la physiologie conçue comme un chapitre du mécanisme universel. Nul besoin de rappeler ici l'épisode de la glande pinéale et des «animaux-machines». Cette conception, bien sûr, ne fit jamais l'unanimité; par exemple, l'animisme devait faire un retour en force au XVIII' siècle, avec G.H. Stahl (Cf. F. Duchesneau, La Physiologie des Lumières, chap. 1). Mais la distinction cartésienne des deux substances (étendue et pensante) étend pratiquement son ombre sur toute la période qui nous occupe. En conséquence, il y a maintenant un abîme entre l'idée et la chose représentée par l'idée. Dans la psychologie aristotélicienne, les formes que reçoit l'âme dans la sensation sont les formes (sans leur matière) des objets perçus eux-mêmes. Dans la nouvelle psychologie " rationnelle », les choses relèvent de la res extensa, et les idées, de la res cogitans : rien des premières ne peut passer dans les secondes ; ou plutôt, l'esprit, ses facultés, ses opérations et ses idées ont maintenant une réelle autonomie devant le monde des choses étendues. La nouvelle psychologie retient cependant de l'ancienne la division des facultés actives de l'âme en perception et volonté, ou croyance et désir, ou entendement et volonté. De plus, pour la plupart des auteurs classiques, les rapports entre le langage et la pensée sont demeurés essentiellement les mêmes que chez les anciens : la pensée est déjà là, toute faite, avant son expression; toutes les parties d'une pensée sont simultanément présentes à l'esprit du locuteur, alors que l'expression de la pensée doit, elle, s'ordonner dans le temps. Mais les penseurs des Lumières (en particulier Condillac) seront de plus en plus sensibles aux effets positifs de l'acquisition du langage sur le développement de la pensée.

4) Pour les rationalistes (Descartes, Arnauld, Cordemoy), le langage est l'«une des plus grandes preuves de la raison» (PortRoyal, 1660, p. 27) ; les langues sont l'œuvre et l'instrument de la Raison, une faculté qui nous place «fort au-dessus des bêtes». Tous les commentateurs s'entendent là-dessus. Mais aux empiristes, on attribue souvent ${ }^{20}$ la thèse que « les langues sont l'ouvrage de la

20. Par exemple, selon J.T. ANDRESEN, «François Thurot and the First History of Grammar ", in Historiographia linguistica, V:1/2, 1978, p. 49; Rüdiger SCHREYER, "Condillac, Mandeville and the Origin of Language », in Historiographia linguistica, 
nature »(Condillac, Grammaire, p. 432). Il me semble que cette façon de voir sous-estime le caractère profondément « rationaliste » de la philosophie sensualiste, et que ce passage de Condillac doit être réinterprété à la lumière de sa philosophie de la Raison et de sa notion de nature.

Les sensualistes, pas moins que les rationalistes et souvent davantage, ont préféré l'autorité de la Raison à celle de la Tradition; eux aussi ont cru aux progrès des "lumières» et ont fait la promotion de la méthode, de la science, de l'éducation, de la tolérance politique, etc. Un examen comparatif des conceptions de la Raison des rationalistes et des empiristes révèle au fond assez peu de divergences. Pour les deux clans, la Raison est une faculté donnée par Dieu aux hommes pour leur permettre de découvrir et de justifier les vérités dans les sciences, et pour choisir les actions qui peuvent le mieux assurer leur salut. La Raison est pour eux une faculté de discernement permettant de distinguer le vrai du faux, le bien du mal, et même le beau du laid. Elle est aussi une faculté de liaison, nous rendant capables de considérer plusieurs individus sous une seule idée générale (l'abstraction), plusieurs idées générales dans un seul jugement, plusieurs jugements dans un seul raisonnement, et plusieurs raisonnements dans une seule théorie ${ }^{21}$. C'est

(même numéro), p. 20, Condillac est supposé rejeter l'idée cartésienne, adoptée par Port-Royal, d'une invention rationnelle du langage. Mais certe affirmation n'est vraie que du premier langage d'action (les expressions naturelles de nos émotions et besoins); elle ne l'est déjà plus pour un langage d'acrion plus développé, comme la danse ou la pantomime, et ne l'est plus du tout pour un langage fait de sons articulés et «choisis», comme le dit bien Condillac. Nous verrons que le premier langage d'action est loin d'être le propre de l'espèce humaine, puisque chaque espèce animale a le sien, et que le mot «langue» ne s'applique proprement quau langage fait de sons articulés, c'est-à-dire fait de « signes d'institution » choisis pour la représentation et la communication des idées. Par ailleurs, R. GRIMSLEY, dans l' «Introduction» de son recueil Sur l'origine du langage, p. 20, affirme que Maine de Biran, parce qu'il «croit que le langage garde toujours un rapport vital avec la raison ", montre par là qu' « il se rapproche... bien plus du rationalisme que de l'empirisme ». Les rationalistes n'ont jamais eu le monopole de la Raison ; les sensualistes avaient eux aussi une conception de la Raison et j'estime que son rôle dans leurs théories de l'origine et de l'usage du langage est loin d'être négligeable.

21. F. Thurot, dans ses Remarques à sa traduction du Hermès de J. Harris (Hermès, ou Recherches philosophiques sur la grammaire universelle, (1796), éd. par A. Joly, Genève-Paris, éd. Droz, 1972), parle d'une «faculté connective et unifiante " (qui est manifestement la Raison) dans les termes que voici: «Au moyen de cette faculté, 
en ce sens que la Raison est une faculté discursive; et ce n'est pas un hasard si les deux principales méthodes rationnelles connues des philosophes et scientifiques de l'âge classique (Descartes les a reprises de Pappos d'Alexandrie) sont l'analyse (qui renvoie au discernement) et la synthèse (qui renvoie à la liaison). Au fond, rationalistes et sensualistes ne divergent sérieusement que sur le statut des notions communes. Pour les rationalistes, la Raison a un contenu qui est fait de principes généraux et abstraits d'une certitude irrévisable, comme "le tout est plus que l'une de ses parties ", "si on retranche une même quantité à deux quantités égales, l'égalité demeure ", etc. Parce que l'induction et l'expérience ne peuvent jamais nous donner de certitude qui égale celle de ces principes, les rationalistes ont conclu que ces connaissances étaient innées. Les empiristes, au contraire, croyaient malgré tout possible de les dériver de l'expérience ; Condillac disait des principes innées de Descartes qu'ils n'étaient que « les proverbes des philosophes ", parce qu'ils présupposent en fait des connaissances déjà acquises.

Une comparaison plus serrée entre Descartes et Condillac est fort révélatrice. Si l'usage normal de la parole constitue, pour Descartes, une preuve de la Raison, c'est que «la raison est un instrument universel qui peut servir en toutes sortes de rencontres " (cf., la cinquième Partie du Discours de la méthode), ou une «faculté illimitée d'innovation adaptée » ${ }^{22}$, et que seule une telle faculté peut nous rendre capables de faire un usage créateur de la parole, c'est-à-dire d'adapter nos discours à n'importe quelle situation nouvelle, et de répondre avec pertinence au sens de n'importe quel discours prononcé en notre présence. Comme l'a bien vu Chomsky, l'usage créateur de la parole est la marque de la

l'esprit considère une idée générale dans plusieurs individus, une seule proposition dans plusieurs idées générales, un syllogisme dans plusieurs propositions; jusqu'à ce qu'enfin, à force de multiplier et d'unir les uns aux autres les syllogismes, comme ils doivent être liés, il s'élève aux régions brillantes et immuables de la science » (p. 378). Soir dir en passant, Thurot est un sensualiste avoué, grand admirateur de Condillac, qu'il considérait comme l'égal des Kepler et Newton; et comme grammairien, il le juge même supérieur à Dumarsais. Cf. F. Thurot, Tableau des progrès de la science grammaticale (Discours préliminaire à Hermès), 1796, éd. par A. Joly, Bordeaux, éd. Ducros, 1970, p. 117

22. Je reprends cette expression de J.-C. Pariente, voir L'analyse du langage à Port-Royal, chap. 2, p. 54. 
Raison parce qu'il est «moralement impossible » de l'expliquer mécaniquement, de la même manière que le comportement des animaux-machines. Sans la Raison, nous serions incapables d'inventions ou d'innovations adaptées aux situations nouvelles. De même, pour Condillac, c'est dans les situations nouvelles que la Raison se met en vedette. Dans son Traité des animaux (1755), il écrit :

La mesure de réflexion que nous avons au-delà de nos habitudes est ce qui constitue notre raison. Les habitudes ne suffisent que lorsque les circonstances sont telles qu'on a qu'à répéter ce qu'on a appris. Mais, s'il faut se conduire d'une manière nouvelle, la réflexion devient nécessaire, comme elle l'a été dans l'origine des habitudes lorsque tout ce que nous faisions était nouveau pour nous. ${ }^{23}$

Un autre point de comparaison intéressant entre Descartes et Condillac concerne la notion de choix rationnel, et il est particulièrement important pour nous puisque la création et l'utilisation des langues requièrent le libre exercice de la volonté (après tout, parler, c'est agir!). Au début de la Quatrième Méditation, Descartes utilise facultas eligendi (faculté d'élire ou de choisir) comme synonyme de «volonté » et de «libre arbitre». Nous agissons librement lorsque la Raison prescrit à la volonté le choix du «meilleur» moyen à prendre (le plus «approprié» dans les circonstances), ou du meilleur plan d'action à adopter, en vue d'une fin. De fait, la liberté d'indifférence représente, aux yeux de Descartes, « le plus bas degré de la liberté » (Quatrième Méditation). De plus, il ne fait aucun doute que pour lui, les expressions linguistiques ont été, à l'origine, instituées par imposition volontaire ; autrement dit, elles ont été choisies comme moyen en vue d'une fin (la communication des pensées) ${ }^{24}$. Le choix des mots peut parfois

23. Dans les Euvres philosophiques de Condillac, vol. 1, p. 363.

24. Descartes écrit (Méditations métaphysiques, Cinquièmes Réponses, Paris, P.U.F., 1974, p. 231): «... les noms ont été pour l'ordinaire imposés par des personnes ignorantes, ce qui fait qu'ils ne conviennent pas toujours assez proprement aux choses qu'ils signifient "; et dans les Principes de Philosophie (OEuvres philosophiques de Descartes, Tome III, éd. Garnier Frères, Paris 1973. Première partie, \# 74) : « $\grave{A}$ peine saurions-nous concevoir aucune chose si distinctement que nous séparions entièrement ce que nous concevons d'avec les paroles qui avaient été choisies pour l'exprimer. » Meilleur sera le choix des mots, plus parfaite sera la langue. L'algèbre représente, pour les philosophes classiques, l'exemple le plus achevé d'une langue bien faite, d'une langue dont les signes ont été choisis avec soin. Ce n'est que par la Raison que les 
être fondé sur de mauvaises raisons, mais il n'est jamais gratuit. Si on peut dire que les langues sont l'œuvre de la Raison, c'est avant tout parce que les mots ont fait l'objet d'un choix rationnel; les notions communes ou idées innées n'ont rien à y voir. De même, chez Condillac, la notion de choix est analytiquement liée à celle de volonté : dans le Traité des animaux, il explique que la phrase "Je veux » ne signifie pas seulement qu'une chose m'est agréable, mais encore qu'elle est l'objet de mon choix ${ }^{25}$. Et lorsqu'il explique, dans sa Grammaire (1775), pourquoi il préfère parler de signes "artificiels » plutôt que de signes «arbitraires", il écrit ceci :

En effet, qu'est-ce au fond que des signes arbitraires? Des signes choisis sans raison et par caprice. Ils ne seroient donc pas entendus. Au contraire, des signes artificiels sont des signes dont le choix est fondé en raison : ils doivent être imaginés avec tel art, que l'intelligence en soit préparée par les signes qui sont connus ${ }^{26}$.

Un peu plus loin, il ajoute:

On se trompe donc, lorsqu'on pense que dans l'origine des langues, les hommes ont pu choisir indifféremment tel ou tel mot pour être le signe d'une idée. En effet, comment, avec une telle conduite, se seroient-ils entendus? 27

Les notions communes ou innées ne jouent donc aucun rôle dans la formation des langues. La rationalité qui est en cause dans la formation des langues et l'usage normal de la parole est une rationalité de type délibératif. Et les principes qui sont invoqués par les philosophes classiques pour expliquer l'invention des

langues peuvent se perfectionner, parce que seule la Raison rend possible un quelconque progrès dans les choses humaines, par le choix de moyens plus appropriés aux fins poursuivies.

25. Op. cit., p. 378. Il ajoute: «Le droit de choisir, la liberté n'appartient donc quà la réflexion»; et la Raison, je le rappelle, est cette « mesure de réflexion que nous avons au-delà de nos habitudes». Il est intéressant de comparer ce passage avec ce que dit Rousseau, dans le Discours sur l'origine de l'inégalité..., lorsqu'il discute de la Raison et de l'origine des langues : «... dans la puissance de vouloir ou plutôt de choisir, et dans le sentiment de certe puissance, on ne trouve que des actes purement spirituels, dont on n'explique rien par des lois de la mécanique. „Plus bas, dans la même page, Rousseau parle aussi de "la faculté de se perfectionner... "; voir $D u$ Contrat social. Discours sur les sciences et les arts. Discours sur l'origine de l'inégalité parmi les hommes, Paris, Union Générale d'Édirions, 1963, p. 265

26. Dans les CEuvres philosophiques de Condillac, vol. 1, p. 429

27. Ibid., p. 431. 
signes d'institution ne sont pas des principes abstraits qui ne peuvent être dérivés de l'expérience, mais simplement des maximes de choix rationel, qui prescrivent de faire le choix du meilleur moyen à prendre en vue d'une fin (c'est-à-dire, le plus approprié, le plus efficace et le moins coûteux étant données les circonstances et les informations dont on dispose), ou de choisir le meilleur plan d'action à adopter en vue d'une fin, selon que ce plan d'action nous offre plus d'opportunités qu'un autre ou qu'il a plus de chances de succès. Ces principes de sens commun ont donné lieu à plusieurs études intéressantes, en particulier au cours de la dernière décennie (par exemple, Simon (1957), Watkins (1970), Rawls (1971), Richards (1971), Grice (1975), Kasher (1976), Harsanyi (1976) et Elster (1979). Ces principes de rationalité sont très souvent utilisés tacitement par les philosophes classiques pour expliquer la formation et l'évolution des langues, le choix des mots et des paradigmes de flexions pouvant alors être justifié par des raisons de commodité, d'économie, de simplicité, d'efficacité et d'élégance. Ils jouent également un grand rôle dans la formulation des règles rhétoriques visant à maximiser l'efficacité de la communication. C'est aussi à l'aide de tels principes que les grammairiens philosophes expliquaient pourquoi il y a, dans la plupart des langues qu'ils connaissaient, des adverbes, des verbes « adjectifs» et des pronoms. En effet, tous s'entendaient pour dire qu'on pouvait éliminer les adverbes, sans perte de sens, en les remplaçant par des syntagmes prépositionnels (par exemple: «sagement»= «avec sagesse»); qu'on pouvait faire de même avec les verbes adjectifs ( «aime» = «est aimant»); et enfin, que les pronoms se définissent par leur capacité à tenir lieu d'un nom. En conséquence, ces trois catégories d'expression ne sont pas nécessaires à la représentation complète de la pensée dans la langue (théoriquement, on pourrait partout s'en passer). Mais les langues sont faites pour communiquer et la communication a ses exigences. Il vaut mieux être bref et économe de ses moyens que prolixe et ennuyeux ! Les adverbes et les verbes adjectifs permettent une économie discursive qui n'est pas amoindrie par une perte d'efficacité dans la communication. Leur invention et leur usage sont donc en accord avec les canons de la raison pratique. De même, les Messieurs de Port-Royal nous demandent d'imaginer ce que serait une conversation où les locuteurs seraient constamment obligés de se nommer au lieu de dire simplement «e »... 
Un dernier point : lorsque Condillac affirme que « les langues sont l'ouvrage de la nature ", il me paraît clair qu'il ne s'exprime pas littéralement. Dans son Dictionnaire des synonymes, aux articles «Langage » et "Langue », il explique qu'il y a deux types de langage : d'abord le langage d'action, fait de gestes, de cris inarticulés, de mouvements des yeux, de la tête, etc., et ensuite, le langage des sons articulés. Le premier n'est pas le propre de l'espèce humaine ; bien au contraire, chaque espèce animale a le sien! Mais le langage des sons articulés s'appelle proprement langue, et les langues sont faites de signes d'institution choisis pour y attacher certaines idées, comme il le disait déjà dans l'Essai sur l'origine des connoissances bumaines de 1746 (première partie, chap. IV). Chez Condillac, ce qui est proprement linguistique, tout ce qui concerne les signes d'institution ou l'expression conventionnelle des idées, dépend de la réflexion et du choix. Pour Condillac, le mot «nature» veut d'abord dire : "premier état d'une chose », et par extension, tout ce qui ne s'explique que par les lois de la mécanique (cf. son Dictionnaire des synonymes, articles «Nature» et «Naturel»). Et dans sa Grammaire (1775, op. cit., p. 431), il dit que

si nous sommes conformés pour parler le langage d'action, nous le sommes également pour parler le langage des sons articulés. Mais ici la nature nous laisse presque tout faire. Cependant, elle nous guide encore. C'est d'après son impulsion que nous choisissons les premiers sons articulés, et c'est d'après l'analogie que nous en inventons d'autres, à mesure que nous en avons besoin. (Je souligne).

La nature nous montre la voie, elle " commence », comme dit Condillac; et c'est ce qu'elle fait avec le premier langage d'action. Mais dès que celui-ci s'enrichit de signes d'institution où il entre du choix, la réflexion et la Raison deviennent nécessaires. Par ailleurs, l'idée que les mots résultent d'un choix ou d'une imposition volontaire (volontary imposition) se trouve aussi chez Locke, le père du sensualisme moderne. Elle n'est donc pas réservée aux seuls rationalistes. À l'inverse, l'idée que les cris inarticulés, les grimaces, mouvements des membres, des yeux, etc., constituent "la première des langues "se trouve dans le Discours physique de la parole (1666) du cartésien Gérauld de Cordemoy ; mais ce n'est là encore qu'une façon de parler (métaphoriquement ou par extension), car l'auteur distingue aussitôt ces signes naturels des signes d'institution, et affirme que ces derniers seraient impossibles sans l'intervention de la Raison. Les mots « langage » et « langue » 
sont parfois utilisés par les rationalistes et les sensualistes pour désigner toute forme d'expression (naturelle ou conventionnelle) de nos pensées; mais, au sens strict, langue concerne l'expression conventionnelle des pensées. La thèse voulant que les langues soient l'ouvrage de la Raison peut donc être attribuée également aux sensualistes, dans la mesure où ils cherchent à dépasser la thèse de l'origine divine du langage et distinguent clairement les signes naturels des signes d'institution.

5) Le principe d'analogie est l'un des plus souvent invoqués par les grammairiens philosophes. Il intervient, par exemple, dans la genèse des noms communs ("généraux ») chez les sensualistes (Locke et Condillac); c'est lui qui, selon Condillac, guide le choix des mots tout au long de la formation et de l'évolution des langues; et c'est lui aussi qui rend compte du mécanisme des métaphores. L'analogie est une sorte de raisonnement qui consiste à établir une proportion entre plusieurs termes en découvrant le terme manquant. Pour y arriver, on doit bien sûr utiliser les termes déjà donnés. Ce mécanisme, si bien décrit par de Saussure (voir les chapitres IV et $\mathrm{V}$ du Cours de linguistique générale), les philosophes classiques l'ont connu et bien mis en valeur. Ainsi, d'après Locke, les premiers mots devaient être des noms propres utilisés pour désigner un seul objet perçu; par la suite, constatant les ressemblances entre les premiers objets nommés et les objets de la même espèce, les hommes ont tout naturellement étendu la signification des noms propres afin qu'ils désignassent également tous les objets de la même espèce, les noms propres devenant ainsi, de proche en proche, des noms communs, signes des idées générales. Ici la première nomination sert de modèle pour les autres: un objet ressemblant fortement à celui de la première nomination sera nommé de la même façon. (Leibniz s'opposait à cette vue, prétendant au contraire que les premiers noms propres avaient souvent un contenu descriptif général). Le choix des premiers mots, nous l'avons vu, ne devait pas être «arbitraire», mais «fondé en raison»; dans leur théorie de l'origine des langues, les philosophes classiques font souvent intervenir la ressemblance son-chose : le mot (le son) aura plus de chance d'être entendu s'il évoque déjà la chose qu'il nomme. Les enfants utilisent souvent ce procédé lorsqu' ils ignorent « le mot juste»; ils remplacent alors le nom de la chose par le son caractéristique qu'elle produit. Toutes 
les langues ont des mots formés de cette façon; par exemple, en français, nous disons que les chats " ronronnent », que les crapauds " croassent », etc. Leibniz croyait qu'Adam avait nommé les animaux du Paradis Terrestre en procédant de cette manière. Par ailleurs, si on veut introduire un néologisme dans la langue, on essaiera autant que possible de le faire de façon à ce qu'il soit compris aisément par les locuteurs compétents de la langue, sans qu'on ait même besoin d'avoir recours à une définition explicite (ce que de Saussure appelle l' " arbitraire relatif»). Par exemple, le féminin de «recteur» sera "rectrice», et non quelque chose comme «blitigri ». Si on veut exprimer la même idée diversement modifiée, la meilleure façon de s'y prendre (la plus économique), c'est de modifier légèrement le même mot (le même radical) plutôt que de créer à chaque fois un mot nouveau et distinct; par exemple, les mots «amour», «aimer», «aimant», «amoureusement», etc., expriment la même idée, mais chaque fois modifiée par des idées accessoires, qui s'ajoutent à la signification principale d'un mot pour la modifier. Pour les grammairiens classiques, la signification complète d'un mot est composée d'abord d'une idée principale, et puis de certaines idées accessoires qui peuvent être exprimées soit par des morphèmes (qui marquent alors la signification « formelle » du mot, s'il signifie à la manière d'un nom, d'un adjectif, d'un verbe, d'un adverbe, etc.), soit par l'intonation ou l'expression du visage au moment de l'énonciation. Enfin, certaines idées accessoires peuvent se joindre (régulièrement ou à l'occasion) à la signification principale des mots et les faire paraître quelquefois «injurieux, civils, aigres, honnêtes », etc. (Voir les chapitres XIV et XV de la Logique ou l'art de penser d'Arnauld et Nicole). Les phénomènes analogiques concernent surtout les « significations formelles »; un mot étranger nouvellement introduit dans une langue prendra tout naturellement les formes nominales, adjectivales, verbales, adverbiales, etc., de cette langue (comme "tripper », "trippatif », etc.) ; il sera décliné et conjugué selon les formes que lui impose sa langue adoptive. C'est le respect de ces modèles, de ces formes, qui donne de la consistance à une langue et tend à réduire le nombre des irrégularités qui la rendent plus difficile. Le principe d'analogie, qui permet aussi d'expliquer le mécanisme des métaphores, est l'un des plus fondamentaux dans l'analyse du langage à l'âge classique. 
6) Il y a des universaux linguistiques substantiels. Partant d'une analyse de la proposition en Sujet-Couple-Prédicat (ou Sujet-Prédicat), les grammairiens philosophes estimaient, suivant en cela une longue tradition remontant à Platon et Aristote, que toutes les langues devaient disposer de moyens pour "marquer », d'abord, les objets que nous concevons; ensuite, ce que nous affirmons ou nions de ces objets; et enfin, l'action de notre esprit qui affirme, nie, conjoint, interroge, désire, prie, commande, doute, etc. Une langue qui ne disposerait pas de ces moyens serait, à cause de son incomplétude expressive, un bien piètre outil de communication. Les grammairiens classiques étaient convaincus que toutes les langues «policées» ou ayant atteint une certaine maturité dans leur développement, devaient avoir des noms communs (appellatifs) et des déterminants (articles, adjectifs), ainsi que des prépositions ou des cas, pour désigner les objets que nous concevons et les relations possibles entre ces objets; elles devaient aussi avoir, pour représenter complètement la pensée, des adjectifs ou des participes pour marquer ce que nous affirmons ou nions des objets conçus; enfin, le verbe «substantif », le verbe "être ", leur paraissaît nécessaire dans toutes les langues pour marquer l'action de notre esprit qui unit les deux termes d'une proposition. (Le nombre et la nature des parties du discours qui sont nécessaires et/ou suffisantes pour la représentation complète de la pensée dans la langue varient quelquefois d'un auteur à un autre et ce qui précède n'est qu'une esquisse de ce qu'il faut s'attendre à trouver dans les grammaires générales.)

Afin de protéger ces principes de la falsification, les grammairiens philosophes développeront plusieurs théories ou hypothèses auxiliaires inspirées de ces principes ou de principes qui ne les contredisent pas (la ceinture de protection). Les « hypothèses auxiliaires » dont il est ici question ne concernent pas les instruments de mesures, comme c'est très souvent le cas dans les sciences naturelles. Ces hypothèses auxiliaires proposent néanmoins des réponses à des questions qui peuvent à première vue sembler embarrassantes. Si la pensée est la même partout et pour tous, alors d'où vient la difficulté de traduire? et d'où vient que l'ordre des mots n'est pas le même dans toutes les langues? Si les langues se forment partout sur les mêmes principes, alors d'où vient leur diversité ? Et si la Raison est à l'œuvre dans toutes les langues, 
comment expliquer cette profusion de termes dits «synonymes», qui paraît contredire les principes d'économie et de simplicité de la raison commune (l'efficacité au moindre coût, ne pas multiplier les entités inutilement, etc.) ? et cette profusion de tropes (métaphores, métonymies, synecdoques, ironies, hyperboles, etc.), et d'idiotismes (latinismes, gallicismes, germanismes, anglicismes, etc.) qui se moquent sans cesse des règles de la langue et y introduisent des irrégularités? Les théories de la traduction, de l'inversion et de l'ordre naturel des mots, de l'origine des langues, de la synonymie, des idées accessoires, des tropes et de l'ellipse s'inspirent, à l'âge classique, des enseignements de la Grammaire Générale. En retour, elles répondaient à (ou prévenaient) certaines questions et objections mettant en jeu les principes de la Grammaire Générale.

\section{LA THÉORIE DES MODES VERBAUX \\ DANS LA GRAMMAIRE GÉNÉRALE CLASSIQUE}

Le verbe fut longtemps considéré par les grammairiens occidentaux comme « le mot par excellence ", ou comme «l'âme du discours ». Tous les grammairiens admettent qu'il y a autant de propositions dans une période qu'il y a de verbe (utilisés ou sousentendus). Du point de vue de la morpho-syntaxe, le verbe est porteur d'un système complexe de flexions variées pour marquer la personne, le nombre, le temps et le mode, qui sont les principaux accidents du verbe. Le temps et le mode sont particulièrement importants pour les grammairiens (surtout après Priscien), car ces deux accidents sont propres au verbe, tandis que la personne et le nombre affectent d'autres parties du discours (comme les pronoms). Mais le mode semble jouir d'une certaine primauté sur le temps et les autres accidents du verbe. En effet, le mode détermine les autres accidents du verbe sans être de la même manière déterminé par eux. Ainsi, le fait qu'un verbe soit à l'indicatif, au subjonctif ou à l'impératif, détermine le nombre des temps dont il est susceptible (en français : huit temps à l'indicatif, quatre au subjonctif et deux à l'impératif); de la même façon, un verbe n'admettra pas le même nombre de personnes suivant qu'il est à l'indicatif ou à l'impératif ; et de même encore pour le nombre (singulier ou pluriel), car c'est le mode qui détermine si le verbe doit ou non en porter la marque (c'est le cas dans tous les modes, sauf l'infinitif). De plus, comme le remarque Beauzée dans l'article «Mode» de l'Encyclopédie, les 
modes, davantage que le temps et les autres accidents, « semblent tenir de plus près aux vues de la Grammaire, ou du moins aux vues de celui qui parle» ${ }^{28}$; en d'autres mots, les modes tiennent davantage à l'intentionnalité du sujet parlant ${ }^{29}$. La très grande majorité des grammairiens occidentaux, de Denys de Thrace (II ${ }^{\mathrm{e}}$ siècle avant J.C.) à André Martinet (Syntaxe générale, 1985), reconnaissent que les modes servent à exprimer conventionnellement certaines « inclinations de l'âme » (Denys de Thrace utilisait enklisis ${ }^{30}$ pour désigner aussi bien les modes que ce qu'ils servent à exprimer, et Priscien, inclinationes animi); les grammairiens de Port-Royal parlaient dans le même sens des "actions de nostre esprit » ou des «mouvements de l'âme»; Dumarsais, qui reprend sur ce point le vocabulaire des Messieurs, parle aussi de certaines «vues » ou «considérations particulières de l'esprit "; les grammairiens anglais des Lumières (Harris, Monboddo et Gregory) associent aux modes certaines energies of the mind, ou des operations of the mind; chez Beauzée, ce sont certaines idées accessoires qui distinguent les modes entre eux et ces idées accessoires se rapportent aux états de l'âme du locuteur. De nos jours encore, Martinet, qui ne consacre aux modes qu'un très court passage, écrit tout de même qu'ils servent à rendre explicite « une prise de position du locuteur par rapport à l'action» ${ }^{31}$. C'est surtout dans leur théorie des modes verbaux que les grammairiens philosophes feront l'étude des procédés que nous utilisons pour exprimer, dans les langues humaines, les attitudes propositionnelles (croyance, désir, doute, souhait, etc.). La théorie générale des

28. N. BeAuZÉE, art. «Mode », in Encyclopédie, ou Dictionnaire raisonnée des sciences, des arts et des métiers, par Diderot et d'Alembert, Tome vingt-deuxième (édition conforme à celle de Pellet, in quarto, à Berne et Lausanne, chez Les Sociétés Typographiques, 1780), p. 8.

29. Comme le remarque $S$. AURoux, dans «Actes de pensée et actes linguistiques dans la Grammaire Générale », in Histoire. Épistémologie. Langage, VIII : 2, 1986. Soit dit en passant, le père de la phénoménologie déclarait, à la toute fin de la Quatrième Recherche logique, prendre « fait er cause pour la vieille doctrine d'une "grammaire générale et raisonnée", d'une grammaire "philosophique" »; Husserl attribue "au rationalisme du XVII e er du XVIII e siècles » l'idée d'une telle grammaire universelle. Cf. Recherches logiques, Tome second, Paris, P.U.F., 1962, PP. 132-133. MerleauPonty ne s'y est pas trompé, cf. «Sur la phénoménologie du langage», in Éloge de la philosophie, et aurres essais. Paris, Gallimard, 1953 et 1960. Pp. 83-84.

30. Cf. G. Nuchelmans, Theories of Propositions (1973), op. cit., p. 101.

31. A. Martinet, Syntaxe générale, Paris, Coll. U, Armand Colin, 1985, PP. 134-135. 
modes verbaux fut donc, à l'époque classique, l'un des lieux privilégiés pour la discussion des rapports entre le langage et la pensée.

On peut distinguer deux approches dans la théorie générale des modes des grammairiens philosophes. La première est représentée principalement par Port-Royal, Dumarsais, Harris, Monboddo et Gregory. Pour ces grammairiens, certains énoncés servent conventionnellement à exprimer des jugements catégoriques (comme ceux dont le verbe est à l'indicatif), et d'autres servent par convention à exprimer soit des jugements non catégoriques (conditionnel, subjonctif), soit des actes de pensée autres que le jugement (impératif, interrogatif, optatif, etc.). Les modes sont alors considérés comme des marqueurs d'actes de pensée. Par exemple, Dumarsais (art. "Construction» de l'Encyclopédie) divise la classe de tous les énoncés en propositions (qui servent à exprimer des jugements) et énonciations (qui expriment des actes de pensée autres que les jugements) : ce qui correspond en gros à la distinction entre les énoncés déclaratifs et non déclaratifs. La seconde approche, que j'appelle réductionniste, est représentée principalement par Beauzée, Beattie et Destutt de Tracy; et pour ces auteurs, toutes nos énonciations expriment finalement des jugements. Ainsi, un énoncé bien formé dont le verbe est à l'impératif ou à l'optatif, ou encore un énoncé interrogatif, ne signifie rien de plus, quand au « fond de pensée ", qu'un énoncé dont le verbe principal est à la première personne du singulier de l'indicatif présent, comme «J'ordonne que... » (ou «Je t'ordonne de... » + infinitif), « Je souhaite que..., » et "Je demande + si..." (ou + pronom interrogatif). Les phrases impératives, optatives et interrogatives ne sont que des ellipses de phrases déclaratives dont le verbe principal signifie l'action accomplie par le locuteur qui ordonne, exprime un souhait ou interroge. Cependant, nous verrons que Beauzée s'écarte quelque peu de cette conception; pour lui, les énoncés non déclaratifs et elliptiques, et leur paraphrase déclarative ne seraient pas synonymes; les modes sont marqués par des morphèmes qui expriment certaines idées accessoires et modifient la signification formelle ou spécifique du verbe (la signification du verbe en tant que verbe), lorsque le radical du verbe prend les terminaisons propres à l'indicatif, au subjonctif, etc. Avant d'examiner plus en profondeur ces deux approches, je crois utile de faire un bref retour historique sur les 
conceptions logiques et grammaticales du verbe qui ont marqué la tradition grammaticale en Occident.

Les premiers grammairiens grecs, Denys de Thrace et Apollonios Dyscole, utilisent surtout des critères morphologiques pour définir le verbe, qu'ils nous présentent comme une partie du discours sans inflexion casuelle, admettant des inflexions pour le temps, les personnes, et le nombre, et signifiant l'action et la passion ${ }^{32}$. L'absence de flexion casuelle semble être un critère aussi important que la présence des autres flexions ; sémantiquement, le rôle du verbe consiste à désigner l'action et la passion. Enfin, notons l'absence des modes dans la définition du verbe des grammairiens grecs, les autres accidents du verbe étant mentionnés comme il se doit. Chez Priscien, la définition du verbe est sensiblement différente: elle mentionne toujours l'absence de flexion casuelle, et que la signification principale du verbe est l'action ou la passion qu'il désigne, mais les deux seuls accidents du verbe qu'elle mentionne sont ceux qui lui sont propres, soit le temps et le mode : le verbe signifie cum temporibus et modis ${ }^{33}$. Denys de Thrace, Apollonios et Priscien reconnaissaient cinq modes: indicatif, impératif, optatif, subjonctif et infinitif. L'infinitif apparaît comme le cas non marqué, le plus simple, le mode dans lequel tous les autres peuvent se résoudre, ou comme un radical commun exprimant simplement l'action (pragma) sans marque de temps, de nombre ni de personne. Quant aux modes personnels, Apollonios les analyse d'une manière qui rend explicite l'acte illocutoire qu'ils servent à exprimer conventionnellement: aux phrases dont le verbe est à l'indicatif, à l'impératif et à l'optatif, il fait correspondre, respectivement, des phrases à l'indicatif présent commençant par "Il affirme que... », «Il ordonne que... », "Il souhaite que... " 34 . (Nous verrons plus loin que cette conception des rapports entre l'indicatif (ou l'infinitif) et les autres modes personnels revient chez plusieurs grammairiens philosophes, tels Harris, Beattie et Destutt de Tracy).

32. Cf., par exemple, G.L. Bursill-Hall, Speculative Grammar of the Middle Ages. The Doctrine of partes orationis in the Modistae. La Haye-Paris, éd. Mouton, 1971, p. 197.

33. Lbid., p. 198.

34. G. Nuchelmans, Theories of Propositions, op. cit., p. 102. 
Mais bien avant les grammairiens d'Alexandrie, les philosophes d'Athènes avaient déjà exposé plusieurs idées au sujet du verbe. Pour Aristote (et bon nombre de logiciens après lui), «Le verbe est ce qui ajoute à sa propre signification celle du temps ", et il indique toujours quelque chose d'affirmé de quelque autre chose " ${ }^{35}$. Ici les critères sont surtout sémantiques: le verbe a une signification propre, un certain attribut ; il affirme l'existence d'un attribut dans un sujet; et « ajoute à sa propre signification celle du temps ». Par ailleurs, les philosophes (péripatéticiens et stoïciens) développeront des classifications pour les "genres de discours》 (ou modes d'énoncé). Les péripatéticiens, par exemple, distinguaient cinq genres de discours : déclaratif, interrogatif, impératif, déprécatif et vocatif ${ }^{36}$; les stö̈ciens en distinguaient deux fois plus. Ces classifications étaient sans doute connues des grammairiens d'Alexandrie.

La tradition des grammairiens, avec leurs critères morphologiques, et celle des logiciens, avec leurs critères sémantiques, se rencontrent pour la première fois au Moyen âge chez les Modistes ${ }^{37}$. Avec eux le verbe ne désigne plus l'action ou la passion, mais plutôt l'être, le devenir, la succession et le flux ; et s'ils retiennent les traits morphologiques soulignés par les grammairiens (les flexions pour le temps, les modes, le nombre, les personnes et l'absence de flexion casuelle), c'est surtout les aspects sémantiques, la «manière de signifier» du verbe qui les intéressent. Cependant ils ne retiendront pas tels quels les critères des logiciens ; l'affirmation n'est retenue que par Michel de Marbais ${ }^{38}$, et le temps est ramené au rang de simple accident du verbe - comme chez les grammairiens -, et on dit maintenant, comme le faisait déjà Boèce ${ }^{39}$, qu'il est consignifié par le verbe. L'affirmation est remplacée par le critère de la séparation: le verbe signifie, séparément du sujet, une modification ou un changement quelconque dans le sujet. Ce qui distingue le verbe du participe, qui signifie lui aussi le changement

35. Aristote, De l'Interprétation, trad. J. Tricot, Paris, éd. Librairie Philosophique J. Vrin, 1966, p. 81.

36. G. Nuchelmans, Theories of Propositions, op. cit., p. 97 et passim.

37. Cf., BurSill-HaLL, op. cit., p. 196.

38. Ibid., p. 196.

39. Sa définition est citée par BuRSILL-HaLL, op. cit., p. 197 : « Verbum est, quod consignificat tempus... ». 
ou la succession, mais en liaison avec le sujet dont il prend, en raison de sa nature adjectivale, le genre et le nombre. Chez Thomas d'Erfurt, le mode du verbe exprime une qualité particulière de la compositio. La compositio est ce qui annule la séparation mentionnée plus haut entre le sujet et le verbe ; c'est ce qui crée le lien entre les deux parties d'une construction intransitive élémentaire. Les diverses qualités de la compositio sont l'indication, le commandement, le souhait et le doute (qui correspondent, respectivement, à l'indicatif, à l'impératif, à l'optatif et au subjonctif). Les modes verbaux, pour les Modistes, servent donc à exprimer certains états psychologiques sur lesquels se fondent les qualités d'indication, de commandement, etc. ${ }^{40}$

Le plus grand des grammairiens de la Renaissance, Sanctius, niera l'existence des modes, catégorie grammaticale qui lui paraît mal fondée, parce que les grammairiens ne s'entendent pas sur la nature, le nombre, et la dénomination des modes. Ramus avait déjà, avant lui, exprimé les mêmes doutes à leur sujet. Sanctius sera suivi sur ce point par quelques grammairiens, et même, plus tard, par Claude Lancelot, dans sa Métbode pour apprendre facilement et en peude temps la langue latine (1644). Mais dans la Grammaire générale et raisonnée, qu'il écrira avec Arnauld, les modes ont la place qui leur revient dans la théorie du verbe. Périzonius, un commentateur de Sanctius, critiquera l'attitude du Maître espagnol sur la question des modes et introduira l'idée que les modes sont aux verbes ce que les cas sont aux noms; ce qui fait des modes un moyen de construction, et pas seulement un moyen d'exprimer les attitudes du locuteur. Cette idée sera critiquée par les grammairiens philosophes (en particulier Beauzée), car la comparaison entre les flexions casuelles et les modes verbaux ne mène pas très loin: l'indicatif ressemble au nominatif dans la mesure où les deux apparaissent comme des cas "non marqué », non déviants, et le subjonctif retient quelque chose de l'accusatif, parce qu'il sert à former des propositions subordonnées qui font office de compléments d'objet de certains verbes. Mais on voit mal comment poursuivre la comparaison. Certains grammairiens philosophes (Dumarsais, Beauzée) qualifient tout de même d'oblique le mode

40. BurSill-Hall, op. cit., PP. 215 et suiv. 
subjonctif, car ce mode ne sert qu'à former des propositions qui sont des parties d'une proposition complète, tout comme « de la choucroute » dans «Je mange de la choucroute » n'est qu'une partie de l'attribut (l'accusatif est un cas oblique). L'idée que les modes sont des moyens de construction vaut d'ailleurs surtout pour le subjonctif, qui marque la subordination. Une autre innovation de la Renaissance est l'ajout d'un mode « potentiel " par le grammairien anglais Thomas Linacre, et ce mode serait marqué, en anglais, par les verbes auxiliaires "may», «might» ou «should» ${ }^{41}$. (Nous verrons plus loin que cette idée sera reprise par l'écossais $\mathrm{J}$. Beattie).

Nous pouvons maintenant examiner les deux approches mentionnées plus haut. La première, qui me semble la plus répandue, associe directement aux différents modes différents actes de pensée; nos diverses énonciations n'expriment donc pas toujours des jugements. La seconde approche associe directement aux modes certaines idées accessoires qui modifient la signification formelle du verbe, en plus d'être l'indice d'une ellipse; il suffit de combler l'ellipse pour faire apparaître le jugement qui se cache derrière toutes nos énonciations.

A) Les modes en tant que marqueurs des actes de pensée. Les logiciens divisaient les parties du discours en catégorèmes et syncatégorèmes; les grammairiens, eux, en mots déclinables et indéclinables. Le grand Arnauld, quant à lui, les divise en deux groupes: il y a les mots «qui signifie les objets des pensées, \& les autres la forme ou la manière de nos pensées "(Grammaire générale et raisonnée, p. 30). Les noms, articles, pronoms, participes, prépositions et adverbes, appartiennent au premier groupe; les verbes, conjonctions et interjections, au second. Les interjections servent à exprimer des émotions (surprise, stupéfaction, horreur, etc.) qui peuvent être considérées comme une forme ou manière de nos pensées, au même titre que le jugement, le désir, l'interrogation, le commandement, et autres « mouvements de l'âme ». Les conjonctions font parties du même groupe parce qu'elles indiquent des opérations de l'esprit (conjonction, disjonction, implication) qui

41. Cf. Ian MiCHaEL, English Grammatical Categories and the Tradition to 1800, Cambridge, C.U.P., 1970, pP. 115 et 425 . 
déterminent la forme logique de nos pensées. Les pronoms interrogatifs servent aussi à marquer un mouvement de l'âme, le désir de savoir, mais les Messieurs rangent les pronoms dans le premier groupe, car leur principale fonction est de marquer confusément les objets de nos pensées.

Il y a enfin le verbe, que Lancelot et Arnauld définissent ainsi : «un mot dont le principal usage est de signifier l'affirmation : c'est à dire de marquer que le discours où ce mot est employé, est le discours d'un homme qui ne conçoit pas seulement les choses, mais qui en juge \& qui les affirme » (G.G.R., p. 95). Il y a quelque chose d'un peu étrange dans cette définition: d'une part, on présente l'affirmation comme le trait essentiel du verbe (après tout, il s'agit d'une définition), mais d'autre part, marquer l'affirmation n'est que son principal usage. Qu'arrive- $t$-il au verbe lorsqu'il ne marque pas l'affirmation? La réponse des Messieurs, c'est «que l'on s'en sert encore pour signifier d'autres mouvemens de nostre ame; comme désirer, prier, commander, \&c. Mais ce n'est qu'en changeant d'inflexion et de Mode » (ibid., pp. 95-96). Le commandement et la prière ne semblent pas à leur place dans une énumération des actions de notre esprit ou des mouvements de l'âme, dans la mesure où ces actes présupposent en général une autre personne et ne semblent pas pouvoir se réduire à de simples «actions de l'esprit » d'une personne seule et isolée. Le caractère «social» de ces actes de pensée sera plus tard reconnu par les common sense philosophers écossais. De toute manière, la «signification principale » du verbe, pour les Messieurs, «est celle qu'il a à l'indicatif ». Mais il faut tout de suite noter que le verbe ne signifie pas l'affirmation de la même manière que les mots affirmatio ou "affirmation", ou les phrases affirmo ou «J'affirme». Le verbe signifie l'affirmation accomplie par le locuteur (actus exercitus), tandis que affirmatio ou affirmo signifient une affirmation conçue par le locuteur (actus significatus). Ainsi, en énonçant Petrus affirmat, un locuteur accomplit une affirmation qui est marquée par la copule implicitement contenue dans le verbe adjectif: Petrus est affirmans, tandis que le participe signifie une affirmation conçue par le locuteur et par lui attribuée à Pierre ${ }^{42}$.

42. G. Nuchelmans, Judgment and Proposition, op. cit., attire l'attention, à propos de cette exemple d'Arnauld et Lancelot, sur la distinction médiévale actus exercitus/actus significatus, Pp. 100 et suiv. 
Avec le critère de l'affirmation, les grammairiens de PortRoyal semblent revenir à la définition du verbe des logiciens. En fait, Arnauld et Lancelot ne font que quelques pas en cette direction ; les grammairiens philosophes des Lumières (Dumarsais, Harris, Beauzée, Condillac, Desturt de Tracy, etc.) iront plus loin sur cette voie. Dans la Grammaire de Port-Royal, le verbe perd deux des fonctions que lui reconnaissait Aristote: signifier un attribut, et indiquer le temps. L'attribut et le temps ne sont plus que des "significations ajoutées ", ajoutées à la signification principale du verbe qui est de marquer l'affirmation. Pour cette raison, le verbe, sous sa forme la plus pure, est le verbe "être» tel qu'utilisé dans les propositions éternelles, à la troisième personne du singulier de l'indicatif présent, comme dans : «Le tout est plus que l'une de ses parties", "La somme des angles intérieurs d'un triangle est égale à deux droits ", etc. Nous avons vu que les autres verbes, les verbes dits « adjectifs», sont des contractions du verbe «être » et d'un parricipe.

Ainsi, les verbes, dans la Grammaire de Port-Royal, servent à exprimer l'action de notre esprit, ou la «manière de nos pensées ». Plus précisément, c'est le mode du verbe qui remplit cette fonction. L'affirmation (ou le jugement) est la principale manière de nos pensées, mais l'interrogation, le commandement, la prière, le simple désir, la concession, en sont tout autant. Ces dernières sont exprimées par les modes autres que l'indicatif. Les modes se répartissent finalement en modes de l'entendement et modes de la volonté. L'indicatif et le subjonctif (les Messieurs ne distinguaient pas encore le conditionnel du subjoncrif et traitaient sous ce dernier les formes en - rais) relèvent de l'entendement, tandis que les modes optatif, concessif et impératif relèvent de la volonté. L'indicatif sert à exprimer des «affirmations simples " (catégoriques), et le subjonctif, des affirmations « conditionnées» (pour les formes en - rais) ou " modifiées » (pour le subjonctif proprement dit). Quant aux modes de la volonté, ils dépendent de la manière dont nous voulons les choses : 1) si ce que nous voulons obtenir ne dépend pas de nous, il s'agit d'un souhait et c'est le mode optatif qui s'emploie; 2) lorsqu'on se contente d'accorder une chose même si absolument on ne la désire pas, c'est alors le mode " potentiel » ou « concessif » (modus potentialis, modus concessivus) qui est requis ; 3 ) enfin, lorsque nous voulons quelque chose qui 
dépend d'une personne de qui nous pouvons l'obtenir, en lui adressant une prière ou un ordre, c'est alors l'impératif qui doit être utilisé. Le mode concessif est mentionné même si aucune flexion verbale ne lui correspond ; en français, on utilise le subjonctif à la troisième personne du singulier: "Qu'il le fasse !», "Qu'il dépense !», «Qu'il perde! », etc. Les Messieurs remarquent aussi que l'on commande souvent avec le futur de l'indicatif ( $T u$ ne tueras point! »).

Le verbe signifie donc quelque chose d'autre que l'affirmation (ou le jugement) lorsqu'il est utilisé dans un mode autre que l'indicatif. Ces modes autres que l'indicatif créent, semble-t-il, des contextes intensionnels ${ }^{43}$, en retirant l'affirmation du verbe indicatif. L'affirmation du verbe est alors suspendue et la liaison du sujet et du prédicat n'est plus présentée que comme possible, ou dépendante d'une condition, ou d'un fait contingent ou probable. Le « mouvement de l'âme » exprimé par le mode se rattache alors à la «matière d'un jugement possible » (sujet + prédicat), selon l'expression fort juste de Dominicy ${ }^{44}$. Ainsi, en supposant que «Marche! » et «J'ordonne que tu marches " soient des énoncés équivalents, le contenu de la clausule ( "que tu marches ») n'est pas l'objet d'une affirmation; il n'est que la "matière d'un jugement possible », même si la phrase complète, elle, exprime bien un jugement mais un jugement qui contient maintenant un opérateur modal. Condillac, dans sa Grammaire (1775), illustre assez bien ce point de vue:

Mais si au lieu de dire $t u$ fais, vous faites, je dis, fais, faites, l'affirmation disparaît, et la co-existence de l'attribut avec le sujet, n'est plus énoncée que comme pouvant ou devant être une suite de mon commandement. (P. 472).

(Notez ici la présence des modalités « pouvoir » et « devoir »).

Quant aux modes impersonnels, les participes sont traités comme des «noms adjectifs», parce qu'ils ne retiennent pas l'affirmation du verbe, quoiqu'ils signifient par ailleurs la même

43. Pour un point de vue contemporain sur cette problématique, voyer R. ZUBER, NonDeclarative Sentences, Amsterdam, John Benjamins Publishing Company, 1983.

44. M. Dominicy, La Naissance de la grammaire moderne, Bruxelles, Pierre Mardaga, 1984, p. 167. 
chose que le verbe (hormis le temps et la personne), c'est-à-dire un certain attribut avec des marques pour le nombre et le genre. L'infinitif par contre offre plus d'intérêt, en raison du fait qu'il retient quelquefois l'affirmation ${ }^{45}$, selon Arnauld et Lancelot; mais la plupart du temps, les infinitifs sont des noms qui ne retiennent pas l'affirmation, et ne portent pas les indications de nombre, de personne et de temps.

Avant la période de l'Encyclopédie (avec Dumarsais et Beauzée), les innovations les plus importantes dans la théorie des modes concernent le conditionnel. Buffier (1709), et plus tard Restaut (1730), font du conditionnel un temps de l'indicatif, qui deviendra avec Destutt de Tracy un “imparfait des temps à venir» (Grammaire, 1803, pp. 209-210). Girard (1747) sera le premier à faire du conditionnel un mode à part, qu'il appelle aussi mode «suppositif».

$$
* * *
$$

Avec Dumarsais, le verbe reçoit une définition qui est neutre eu égard aux modes. Le grammairien attitré de la grande Encyclopédie ne dit pas que le verbe sert à marquer l'affirmation (ce qu'il ne fait proprement qu'à l'indicatif), mais plutôt l'énonciation (ou l'action de l'esprit) et l'existence (réelle ou imaginée) du sujet sous telle ou telle qualification. La théorie du verbe de Dumarsais est bien plus proche de l'analyse d'Aristote que celle de Port-Royal ${ }^{46}$. Il adopte une analyse bipartite de la proposition où le verbe constitue une partie essentielle du prédicat, car c'est lui qui indique que quelque chose est dit de quelque chose d'autre. Le verbe signifie de plus l'existence (réelle ou imaginée) d'un certain

45. ARnauld et LanCelot, Grammaire générale et raisonnée, Stuttgart-Bad Cannstatt, Friedrich Frommann, 1966, Pp. 116 er suiv. Selon les Messieurs de Port-Royal, l'infinitif joue quelquefois le rôle d'un pronom relatif en liant deux propositions, comme dans Scio malum esse fugiendum: "Scio" fait seule une proposition, et "malum est fugiendum" en fait une autre. L'infinitif «esse» fait en sorte que la seconde proposition devienne une partie de la première.

46. Cf. Gunvor Sahun, César Chesneau Du Marsais et son rôle dans l'évolution de la grammaire générale, Paris, P.U.F., 1928, p. 310. 
attribut dans un sujet. Dans une phrase comme «Dieu est toutpuissant », ce n'est pas seulement «tout-puissant » qui est jugé de Dieu, mais qu'Il est tout-puissant. C'était revenir à l'idée des logiciens et grammairiens grecs que le verbe «être » est le verbe d'existence, idée qui fut perdue lorsque Priscien traduisit "byparktikon rhéma», par «verbum substantivum». Cette théorie du verbe "être » comme verbe d'existence se répandra au XVIII siècle chez les grammairiens philosophes, aussi bien en France qu'en Angleterre.

Pour Dumarsais, le verbe est donc un mot qui «marque l'action de l'esprit qui adapte ou applique un qualificatif à un sujet, de quelque manière que cette application se fasse" (cf. art. "Construction» de l'Encyclopédie). L'application d'un qualificatif à un sujet peut se faire sous la forme de l'affirmation, ou sous la forme du commandement, du souhait, de la condition ou de la dépendance. La définition de Dumarsais, contrairement à celle de Port-Royal, embrasse tous les emplois du verbe, peu importe le mode. Ce qui donne lieu chez lui à une division de toutes les "propositions considérées grammaticalement en propositions proprement dites, qui servent à exprimer des jugements, et en énonciations, qui servent à exprimer des actions de l'esprit autres que le jugement :

Voilà une différence essentielle entre les propositions : les unes sont directement affirmatives ou négatives, \& énoncent des jugements ; les autres n'entrent dans le discours que pour y énoncer certaines vues de l'esprit ; elles peuvent être appelées simplement énonciations.

Tous les modes du verbe, autres que l'indicatif, nous donnent ces sortes d'énonciations, même l'infinitif, sur-tout en latin. (Art. «Construction », p. 184).

Les modes sont donc différentes formes verbales « appropriées aux divers besoins de l'énonciation", comme le dira plus tard François Thurot ${ }^{47}$. Les propositions énoncées par le mode indicatif sont appelées «directes», et les énonciations, énoncées par les autres modes, sont appelées « obliques » ou « simples énonciations ». Le rapport entre les modes verbaux autres que l'indicatif et les énoncés non déclaratifs est évident. Pour le reste, Dumarsais a peu

47. F. Thurot, Remarques à sa trad. du Hermès de J. Harris, op. cit., p. 154. 
d'idées originales sur les modes. Sa classification des modes est on ne peut plus traditionnelle (il admet l'indicatif, l'impératif, le subjonctif et l'infinitif, auxquels s'ajoute l'optatif «en certaines langues ", dit-il) ; il ne reconnaît pas encore de mode conditionnel. Il est toutefois l'un des premiers à distinguer entre les modes personnels (ou « finis »), qui expriment l'action de notre esprit et qui entrent dans les propositions et les énonciations, et les modes impersonnels (infinitifs), qui n'expriment pas d'inclinations de l'âme. Cette distinction, et sa conception du subjonctif servant à exprimer la "subordination des idées », semblent représenter des progrès réels sur les conceptions de l'époque ${ }^{48}$.

Jetons maintenant un coup d'œil rapide du côté des grammairiens philosophes anglais, notamment Harris, Monboddo et Gregory.

$$
\text { * * * }
$$

L'ouvrage de James Harris, Hermes, or, a Pbilosophical Inquiry Concerning Language and Universal Grammar (1751), est le premier ouvrage important de Grammaire Générale à paraître en Angleterre. L'influence qu'il a eue sur les grammairiens anglais se compare à celle de la Grammaire générale et raisonnée du côté des Français. Les positions de Harris concernant le verbe s'apparentent, comme celles de Dumarsais, aux conceptions aristotéliciennes. Le verbe exprime l'existence d'un attribut dans un sujet. Harris reprend des péripatéticiens la division des facultés actives de l'âme en perception et volonté. Tout discours sert à exprimer un état cognitif ou volitif de l'âme, et les modes verbaux résultent de la diversité des affections de l'âme et des diverses manières de les exprimer. Les modes qui se rapportent à la perception sont l'indicatif, le «potentiel» (qui correspond ici au conditionnel), et le subjonctif; dans le premier cas, la perception de l'âme est affirmée positivement, dans le second, elle l'est d'une manière conditionnelle, et dans le cas du subjonctif, l'affirmation est subordonnée à une affirmation positive. Les modes qui relèvent de la volonté sont l'interrogatif, l'impératif et le déprécatif ; le premier 
sert bien sûr à former des questions ; le second, à commander à un subalterne; et le troisième, à adresser une demande à un supérieur. Pour Harris, les modes se déterminent en grande partie d'après les diverses espèces de propositions ${ }^{49}$. Tous les modes ont en commun une propriété : celle d'exprimer les «affections de l'âme»; c'est pourquoi l'infinitif n'est pas un véritable mode. Harris fait quelques observations intéressantes sur la pragmatique des modes verbaux, en particulier sur les modes interrogatif, impératif et déprécatif. Ceux-ci, contrairement aux modes indicatif, potentiel et subjonctif, exigent toujours une réponse, qui peut être soit une proposition, soit une action. La direction d'ajustement de l'énonciation entre ici en ligne de compte. L'interrogatif exige toujours une proposition en guise de réponse; mais la réponse au mode de demande (impératif et déprécatif) peut à l'occasion être une simple action. Harris distingue, comme on le fait aujourd'hui, deux types de questions : les questions qu'il appelle «simples» et «définies", auxquelles on peut répondre par « oui » ou « non », et les questions " complexes» et « indéfinies », auxquelles on doit répondre par une proposition (complète ou elliptique). Enfin, Harris observe comme beaucoup d'autres que tous les modes personnels peuvent se résoudre par l'infinitif et l'indicatif (par exemple: ambulo = indico me ambulare, etc.) ; il observe aussi (comme Apollonios et Priscien) que l'infinitif a une certaine affinité avec tous les verbes impliquant une certaine tension psychologique (désir et volonté), verbes que Priscien appelait verba volontiva; on peut dire, par exemple, «Je veux vivre», mais pas «Je mange vivre », etc. Dans la théorie des actes de parole, on peut constater de même que les actes de types directif et « engageant 》 (commissive) sont exprimés par des énoncés dont le verbe illocutoire accepte, comme complément d'objet, une clausule infinitive: «Je t'ordonne de marcher », «Je m'engage à rester $\gg$, etc.

$$
* * * *
$$

49. J. Harris, Hermès, ou Recherches philosophiques sur la grammaire universelle (Remarques et trad. de F. Thurot, avec une intr. de A. Joly). éd. par A. Joly, GenèveParis, Librairie Droz, 1972, p. 136, note 2. 
James Burnett, alias Lord Monboddo, fit paraître à partir de 1773 un imposant ouvrage en plusieurs volumes intitulé : Of the Origin and Progress of Language. L'ouvre de Monboddo fut assez influente, tant en Angleterre qu'à l'étranger, surtout en Allemagne où Herder la fit connaître en même temps que celle de Harris. Pour Monboddo, la première chose à considérer dans le verbe est l'expression de ce qu'il appelle «an energy of the mind of the speaker », c'est-à-dire, soit une affirmation (assertion), soit une volition. Les volitions se divisent en souhait et commandement. Tous les verbes servent à exprimer une affirmation, un souhait ou un commandement. Monboddo place l'interrogation dans la catégorie des souhaits, car celui qui interroge, dit-il, souhaite ou désire être informé. Notons que dans la théorie des actes de parole, les questions font aussi partie des actes directifs (les questions sont des requêtes) qui ont le désir pour condition de sincérité. L'expression d'un attribut et de l'existence de cet attribut dans un sujet sont également essentielles au verbe, «for when we affirm any thing, we assert that it does exist ; when we command it, we desire that it should exist ; and when we wish fot it, it is that it may exist ${ }^{50}$. Monboddo critique les définitions qui font du verbe un mot signifiant l'affirmation (à la manière de Port-Royal), parce que l'affirmation n'est que l'une des «énergies » signifiées par le verbe. Quant aux modes, ils servent justement à exprimer ces «énergies de l'esprit » que sont l'affirmation, le commandement et le souhait. L'affirmation est exprimée par l'indicatif et le subjonctif; le souhait ou la prière, par l'optatif; et le commandement par l'impératif. Monboddo ne reconnaît pas de mode interrogatif ni de mode potentiel, parce qu'ils ne sont pas explicitement marqués par une flexion verbale. L'infinitif n'est pas considéré comme un mode, parce qu'il n'exprime aucune «énergie de l'esprit », comme le font les modes personnels. L'infinitif n'est donc pas, pour lui, un verbe ou une forme verbale, mais un simple substantif.

$$
* * *
$$

50. J. Burnett (Monboddo), Of the Origin and Progress of Language, vol. 1, Menston, Scolar Press, 1967, p. 120. 
Le texte du physicien et grammairien écossais James Gregory, Theory of the Moods of Verbs (1790), représente à mon avis sous sa forme la plus achevée la théorie des modes comme marqueurs d'actes de pensée. De plus, en élargissant le cadre des théories idéationnelles du langage pour y introduire des opérations sociales de l'esprit, il obtient, en sémantique et en pragmatique, des résultats qui rappellent par moment ceux obtenus dans le cadre de la théorie des actes de parole. Gregory s'inspire en partie de Monboddo, mais le critique et va beaucoup plus loin en mettant à profit quelques idées de son ami Thomas Reid.

Pour Monboddo, nous l'avons vu, le mode n'est pas un simple « accident » du verbe, car l'expression d'une energy of the mind fait partie de l'essence du verbe. Par conséquent, l'infinitif ne pouvait être qu'un nom verbal, puisqu'il n'exprime aucune attitude du locuteur. Gregory le critique sur ce point ; comme d'autre grammairiens (Arnauld, Lancelot et Dumarsais entre autres), il remarque qu'en latin surtout, l'infinitif sert souvent, comme verbe, à exprimer des pensées complètes. L'infinitif est donc un verbe sans mode. De cela, il tire la conclusion suivante: "It is only the capacity or susceptibility of mood, that can with propriety be said to be essential to a verb" 51 . Ce qui est essentiel au verbe, c'est de pouvoir accepter tel ou tel mode. De la même façon, ce n'est pas la division, mais la divisibilité qui est essentielle à une ligne géométrique. Il critique aussi Monboddo pour avoir limité indûment le nombre des modes à trois seulement (indicatif, optatif et impératif, le subjonctif se rapportant au premier, et l'interrogatif, au second). Mais Gregory n'insiste pas moins pour ne reconnaître que les modes verbaux explicitement marqués par une flexion verbale caractéristique; ainsi, l'anglais n'a pas de mode interrogatif ou optatif. Toutefois, Gregory utilise en fin de compte la notion de mode grammatical, qui comprend, en plus des modes verbaux, d'autres marques grammaticales, comme l'ordre des mots et les affixes.

L'originalité de Gregory tient justement au fait qu'il distingue soigneusement entre les modes de pensée (moods of thought) et

51. J. GREGORY, Theory of the Moods of Verbs, in Transactions of the Royal Society of Edindurgh, 1790, p. 196. 
les modes grammaticaux (grammatical moods), qui correspondent à deux manières d'envisager les modes: soit relativement à la pensée humaine, soit relativement à une langue particulière. Les modes grammaticaux peuvent varier d'une langue à une autre, mais les modes de pensée sont les mêmes partout et pour tous. Les modes grammaticaux sont les marques qui expriment littéralement les modes de pensées. Les modes grammaticaux sont formellement établis par la grammaire de chaque langue; mais le nombre des modes de pensée excède de beaucoup celui des modes grammaticaux, ce qui nous oblige souvent à utiliser le même mode grammatical pour exprimer différents modes de pensée. Mais les modes de pensée qui ne sont exprimés par aucun mode grammatical caractéristique peuvent néanmoins être signifiés par un énoncé dont le verbe principal est à la première personne du singulier de l'indicatif présent : "Je vous exhorte de... », «Je vous avise de... », etc. Parmi les modes de pensée énumérées par Gregory (environ 35), on retrouve des actes comme affirmer, nier, témoigner, prédire, prophétiser, questionner, répondre, souhaiter, espérer, s'attendre à, croire, savoir, douter, supposer, stipuler, commander, prier, requérir, supplier, aimer, haïr, craindre, désespérer, s'étonner, admirer, avertir, jurer, aviser, refuser, exhorter, dissuader, encourager, promettre, menacer, etc., etc. Plus de la moitié des exemples donnés par Gregory sont des actes illocutoires ; d'autres correspondent soit à des actes perlocutoires (dissuader, encourager, etc.), soit à des attitudes propositionnelles ou états psychologiques (aimer, haïr, admirer, etc.). Ce que Wittgenstein disait des multiples emplois que nous faisons des phrases en parlant, Gregory le disait de ses modes de pensée : ils sont «innombrables » (numberless). Mais sitôt après il ajoute qu'en raison des différences de degré, des nombreuses ressemblances et affinités entre les modes de pensée, il est possible de les ordonner et de les classifier! Tous les actes ou modes de pensée ont en commun quelque chose que Gregory appelle, après Harris et Monboddo, une energy. Ce concept d'énergie est clairement une approximation et une anticipation d'un autre concept «dynamique», celui de «force illocutoire» (Frege, avant Austin, utilisait Kraft).

Ce qui étonne un peu dans l'énumération des modes de pensée de Gregory, c'est la présence d'actes qui présupposent une interaction verbale entre (au moins) deux personnes (commander, 
promettre, supplier, questionner, etc.), et qui ne semblent pas pouvoir se réduire à des simples «opérations de l'esprit» d'un sujet individuel. Mais Gregory reprend de Thomas Reid la distinction entre les opérations solitaires de l'esprit (telles croire, désirer, douter, etc.), qui ne supposent qu'un sujet individuel, et les opérations sociales de l'esprit (commander, promettre, supplier, etc.), qui impliquent une référence mentale à (au moins) une autre personne. Daprès Thomas Reid, toutes les langues sont faites pour exprimer aussi bien les opérations sociales que les opérations solitaires de l'esprit; il dit même que l'expression des opérations sociales de l'esprit « is the primary and direct intention of language. " 52 Des personnes vivant dans la solitude la plus complète n'auraient, selon Reid, aucun besoin d'un langage.

Gregory met clairement en relief la rationalité présente dans la création et l'utilisation des modes grammaticaux. Ces derniers constituent des manières concises et efficaces d'exprimer les modes de pensée les plus fréquents, les plus importants et les plus intéressants. Cela devient évident lorsqu'on compare les phrases marquées par un mode grammatical ( VViens ici!») avec les circonlocutions ou paraphrases qui leur sont équivalentes quant au «au fond de pensée» («Je t'ordonne de venir ici»). Les modes grammaticaux, comme les autres flexions verbales et casuelles, expriment beaucoup mieux que ne le ferait une succession de mots les rapports intimes et les diverses relations entre nos pensées, rapports ou relations qui ne sont pas successifs, mais simultanés dans l'esprit du locuteur. Le mode grammatical est donc plus «naturel », plus « élégant » et plus « énergique » que la paraphrase qu'on peut en donner à la première personne de l'indicatif présent. Par ailleurs, Gregory observe qu'une multiplication des modes grammaticaux rendrait les langues plus difficiles à apprendre, et nuirait finalement à la clarté et à l'efficacité de la communication. On trouve donc des modes grammaticaux dans toutes les langues parce qu'ils sont utiles et efficaces, mais leur nombre doit toujours être assez restreint.

Enfin, Gregory jette les bases de ce que nous appellerions aujourd'hui une « pragmatique des modes ». En effet, il montre, en

52. T. ReID, Essays on the Intellectual Powers of Man, Cambridge (Mass.) et Londres, Presses du M.I.T., 1969, p. 73. 
multipliant les exemples, qu'un mode est souvent mis à la place d'un autre qui exprimerait littéralement le mode de pensée du locuteur. Ce genre de "transfert» ou «transport» d'un mode grammatical à un autre, Gregory le qualifie de «métaphorique » (au sens aristotélicien du terme). Ainsi, au lieu de dire : «Si vous êtes généreux, alors donnez ! 》, on peut dire : «Êtes-vous généreux ? alors donnez!»; de même, au lieu d'utiliser l'impératif pour commander, on peut se servir du futur de l'indicatif : "Tu iras!», ou même de l'interrogatif avec un ton menaçant : «Iras-tu? ». Les «questions rhétoriques » sont aussi de ce genre : « Ne fait-il pas un temps superbe aujourd'hui? » pour: "Il fait un temps superbe aujourd'hui», etc. Cette pragmatique des modes rappelle par moment le traitement des actes de discours indirects proposé par Searle ; mais Gregory, malheureusement, n' indique pas clairement par quelles inférences on doit passer pour arriver à déterminer la «signification du locuteur».

B) L'approche réductionniste des modes verbaux. Si l'approche que nous venons d'examiner présente certaines analogies avec la théorie des actes de parole, ou avec les théories (comme celle d'E. Stenius ${ }^{53}$ ) qui distinguent dans nos énonciations un élément modal (modus) d'un « radical propositionnel » (dictum), l'approche représentée par Beauzée, Beattie et Destutt de Tracy, se compare plutôt aux travaux de ceux qui estiment, comme D. Lewis, qu'une "sémantique sans aucun traitement des conditions de vérité n'est pas une sémantique » ${ }^{54}$. Dans l'approche réductionniste des grammairiens philosophes, toutes les énonciations se réduisent, en dernière analyse, à l'expression de jugements, c'est-à-dire à des énoncés déclaratifs susceptibles d'être vrais ou faux, et qui leur sont équivalents quant au «fond de pensée».

Dans sa Grammaire générale (1767), Beauzée critique longuement la conception du verbe des grammairiens de Port-Royal,

53. Erik Stenius, "Moods and Language Game », in Synthese, 17 (1967), pp. 254-274.

54. D. LEwIS, "General Semantics », in D. Davidson et G. Harman (éd.), Semantics of Natural Language, Dordrecht, Reidel, 1972, p. 169. 
pour finalement développer une conception qui n'est pas sans rappeler celle de Dumarsais: "les verbes sont des mots qui expriment des êtres indéterminés, en les désignant par l'idée précise de l'existence intellectuelle avec relation à un attribut » (Tome 1, p. 402). Les verbes, comme les adjectifs et les participes, désignent des «êtres indéterminés», comme «blanc» désigne tous les êtres indéterminés qui sont blancs, «marche », les êtres indéterminés qui marchent, etc. «L'existence intellectuelle », c'est l'existence qu'a un sujet que je conçois dans mon esprit ; ainsi, un cercle-carré n'a pas d'existence réelle, mais il a une «existence intellectuelle » dans mon esprit lorsque j'affirme: "Un cerclecarré est quelque chose d'impossible». Enfin, cette existence intellectuelle est conçue en relation avec un attribut qui la qualifie de telle ou telle manière. La perception de l'existence intellectuelle d'un sujet avec relation à un attribut n'est rien d'autre, pour Beauzée, que ce que les logiciens appellent « jugement ».

Dans l'article «Proposition » de l'Encyclopédie, Beauzée s'en prend à la distinction de Dumarsais entre proposition et énonciation ${ }^{55}$. Voici comment il résume la perspective qu'il adopte:

Nous parlons pour transmettre aux autres hommes nos connoissances, \& nos connoissances ne sont autre chose que la perception de l'existence intellectuelle des êtres, sous telle ou telle relation, à telle ou telle modification. Si un être a véritablement en soi la relation sous laquelle il existe dans notre esprit, nous en avons une connoissance vraie; s'il n'a pas en soi la relation sous laquelle il existe dans notre esprit, la connoissance que nous en avons est fausse; mais vraie ou fausse, cette connoissance est un jugement, \& l'expression de ce jugement est une proposition (p. 585).

Nous ne parlons que pour transmettre nos connaissances ou informer autrui des perceptions (= jugements) de notre esprit ; et dès que l'esprit perçoit ou considère l'existence intellectuelle d'un être avec telle relation à telle modification, c'est toujours à la manière d'un jugement. Il n'y a donc pas lieu de distinguer différents types de propositions et d'actes de pensée comme le faisait Dumarsais. «Ainsi, conclut-il, il faut convenir qu'il n'y a en effet qu'un jugement qui puisse être le type ou l'objet d'une

55. Pour un examen de cette controverse (posthume) opposant Beauzée à Dumarsais, voir S. Auroux, La Sémiotique des Encyclopédistes, Paris, Payot, 1979, pp. 92 et suiv. 
proposition, \& je conclus qu'il faut dire qu'une proposition est l'expression totale d'un jugement » (ibid.). Plus loin, dans le même article, Beauzée distingue les propositions «expositives » (c'est-àdire déclaratives) des propositions «interrogatives», lesquelles sont toujours elliptiques, et servent à l'expression d'un jugement sur lequel le locuteur a des doutes ou des incertitudes relativement au sujet, à l'attribut, ou à la relation qui les unit.

Le système des modes de Beauzée est exposé dans sa Grammaire générale et dans l'article "Mode» de l'Encyclopédie. Les modes sont soit personnels, soit impersonnels. Seuls les premiers servent à former des propositions, et donc à exprimer des jugements. L'infinitif et le participe appartiennent, respectivement, à la classe des noms et à celle des adjectifs. Les modes personnels se divisent en modes directs (indicatif, impératif et conditionnel), et en modes obliques (subjonctif et optatif). Les modes directs servent à former la proposition principale que l'on veut exprimer. L'indicatif exprime purement et simplement l'existence intellectuelle d'un sujet avec relation à un attribut ; l'impératif le fait en ajoutant à la signification « formelle » ou "spécifique » du verbe 56 l'idée accessoire de la volonté de celui qui parle ; et le suppositif (conditionnel), en ajoutant l'idée accessoire d'une supposition préalable. Le subjonctif est un mode oblique, parce qu'il n'exprime qu'un «jugement accessoire » et «subordonné au principal », et parce qu'il ne sert à former, le plus souvent, que des propositions incidentes (ou subordonnées), qui ne sont qu'une partie d'une proposition complète, comme une sorte d'accusatif. Enfin, les modes se divisent encore en purs et mixtes; les modes purs (indicatif, infinitif et participe), sont ainsi appelés parce qu'ils n'ajoutent aucune idée accessoire et étrangère à la signification formelle ou spécifique du verbe, tandis

56. La signification «formelie 》 ou "spécifique " du verbe s'oppose à sa signification « objective "; la signification objective d'un mot est l'idée de la chose qui lui est associée ; la signification formelle est la manière dont le mot présente sa signification objective à l'esprit des sujers parlants (d'une manière nominale, verbale, adjectivale, adverbiale, etc.). Cette significarion est "spécifique " car elle est celle d'une espèce particulière de mor er est la même pour tous les mots de cette espèce. Ainsi, les mots " amour ", " aimant ", " amoureusement ", " aimé \#, ont la même signification objective (disons, l'idée d'amour), mais différentes significations formelles. Les modes, selon Beauzée, modifient la signification formelle des verbes, et non leur signification objective. 
que les modes impératif, suppositif et subjonctif ajoutent au verbe une idée accessoire particulière qui crée un contexte intensionnel. Les modes mixtes se distinguent ainsi les uns des autres par certaines idées accessoires, « \& il auroit été possible, écrit Beauzée, d'introduire plusieurs autres modes de la même espèce, par exemple, un mode interrogatif, un mode concessif, \&c. » (art. "Mode», p. 11).

M. Auroux ${ }^{57}$ décrit fort bien ce qu'a de «remarquable " la position de Beauzée concernant les phrases interrogatives, optatives, et les phrases impératives à la troisième personne du singulier (ex. : «Qu'il parte immédiatement !»). Ces phrases sont elliptiques, mais «la restitution de l'hyperphrase détruit la modalité", et tranforme une phrase interrogative, optative ou impérative en une phrase qui exprime encore la volonté, le désir ou le souhait, mais à la façon d'une phrase "expositive». Par conséquent, les phrases interrogatives, optatives et impératives ne s'énoncent que sous une forme elliptique, et c'est l'ellipse, non l'hyperphrase ( Je veux que... », «Je souhaite que... », etc.) qui constitue la marque de la modalité. Ces phrases elliptiques et les phrases expositives correspondantes ne sont donc pas synonymes.

$$
\text { * * * }
$$

La seconde partie de l'ouvrage de l'écossais James Beattie (The Theory of Language, 1783) s'intitule: Universal Grammar. Beattie y reprend la définition du verbe qui en fait un mot "necessary in every sentence, and signifiying affirmation 》 (p. 191). Beattie ne dit pas, comme les grammairiens de Port-Royal, que signifier l'affirmation n'est que le principal usage du verbe ; il s'en tient plutôt à la seule affirmation. Par conséquent, tous les énoncés dont le verbe n'est pas à l'indicatif doivent se résoudre par l'indicatif.

Beattie établit une étroite relation entre les différents types d'énoncés simples (simple sentences), et les divers modes verbaux :

Every sentence contains a verb expressed or understood ; and that verb must be in one or other of those forms, which Grammarians call

57. Cf. " Actes de pensée et actes linguistiques dans la Grammaire Générale », op. cit. 
moods. Now every mood has a particular meaning, and gives a peculiar character of the sentence; and, therefore, simple sentences may be divided into as many sorts, as there are supposed to be moods in a verb. (P. 189)

Les énoncés simples à l'indicatif (affirmatifs et négatifs) servent à exprimer les affirmations, comme « He is good », « He is not good». Une phrase dont le verbe est au subjonctif, comme «I know not whether he be good ", se résout, après analyse, en une proposition déclarative : "That he is good (or, his goodness) is to me unknown". Le mode qu'il appelle «potentiel» (potential), et qui est rendu en anglais par des verbes modaux auxiliaires dans " He may be good », ou « He ought to be good", exprime aussi des propositions ou affirmations: "To be good is in his power ", ou "To be good is his duty ". Les phrases optatives, comme «May he be good», contiennent également une affirmation: "That he should be good is what I wish for ». Il en va de même pour des phrases interrogatives, comme «Is he good? », et impératives, comme «Be thou good!", qui se résolvent, respectivement, par «It is my desire to be informed, whether he be good », et «It is my command», ou « it is my entreaty, that thou shouldst be good». (Les exemples sont tous de Beattie). Peu importe le mode du verbe, tous les énoncés simples expriment, après analyse, des affirmations.

$$
* * *
$$

L'idéologue Destutt de Tracy analyse les modes autres que l'indicatif d'une manière très semblable à celle de Beattie. Le premier Tome de ses Éléments d'idéologie s'occupait de l'origine et de la formation des nos idées (l'«Idéologie proprement dite »), tandis que le second, la Grammaire, s'occupe de l'expression de nos idées dans le discours. La Grammaire Générale n'est que «la continuation de la science des idées ». Or, tous nos discours visent au fond le même but: exprimer des jugements, même s'il faut parfois un moment de réflexion pour faire mentir les apparences. "L'essence du discours est donc d'être composé de propositions, d'énoncés de jugements " (Grammaire, p. 33). Mais les langages articulés, dans leur évolution, «ont été si travaillés, si tourmentés, si sophistiqués ; ils ont revêtu des formes si variées, si syncopées, si 
détournées, que l'on a peine à reconnaître, à travers tant de déguisements, en quoi consiste la véritable expression de la pensée» (ibid., pp. 36-37). Au cours des siècles, les peuples ont forgé les instruments indispensables " pour rendre l'expression de la pensée plus complète et plus facile » (ibid., p. 67). Le nombre de nos idées, perceptions et jugements excède de beaucoup le nombre des moyens linguistiques dont on dispose pour les exprimer. Les «systèmes de signes» que sont les langages (une "émission de signes » est un discours) ne sont donc efficaces et viables que dans la mesure où ils permettent de faire beaucoup avec peu. Pas étonnant, souligne l'idéologue, qu'une bonne partie de ce que nous exprimons demeure sous-entendue, que les mots qui reviennent le plus souvent dans le discours (les indéclinables, comme les conjonctions et les prépositions) soient presque toujours, dans toutes les langues, des monosyllabes, et que les mots déclinables aient la capacité d'exprimer plusieurs significations, comme les verbes, qui peuvent exprimer l'attribut, le temps, le nombre, la personne, la voix, l'existence et le jugement, et les noms, qui peuvent exprimer, en plus de leur signification principale, le nombre, le genre et le cas.

Tous nos discours expriment des jugements, quoique souvent sous une forme elliptique; lorsque nous disons : "Je souffre», ou "Je veux », nous exprimons le jugement que cette souffrance, cette volonté, est en nous. Il y a d'ailleurs pour l'idéologue une asymétrie entre le jugement et les autres actes de pensée du point de vue de leur expression : pour exprimer un désir, un souhait, un doute, etc., il suffit de le nommer en disant : «Je veux», "Je souhaite», etc. ; mais pour exprimer un jugement, cela ne suffit pas. Nommer l'acte de juger ce n'est pas l'accomplir. Pour exprimer un jugement, il faut énoncer le sujet, l'attribut, et le signe de l'affirmation. Si on dispose de ces signes, on peut alors exprimer tous les autres actes de pensée simplement en affirmant qu'on les a. C'est le verbe qui marque l'affirmation; ou plus précisément, c'est «la forme du verbe » (ibid., p. 27) qui exprime le jugement, car dans les phrases : "Pierre être grand », «La pêche que je tiens », il y a chaque fois un verbe, mais pas expression d'un jugement. Pour exprimer un jugement, un verbe doit porter la marque de l'un des modes définis (c'est-à-dire personnels). Que seuls les verbes aient des modes se comprend du fait que le verbe marque l'existence et que pour être 
d'une certaine manière (positive, conditionnelle, subordonnée) il faut d'abord et tout simplement exister.

Le verbe est nécessaire dans toutes les propositions; c'est lui qui détermine le sens de la proposition dans laquelle il entre. Les différentes manières de déterminer le sens d'une proposition ont été marquées par différents modes. Pour établir la "véritable valeur de toutes les propositions possibles », il suffit donc d'examiner "les différentes formes (que le verbe) est capable de revêtir» (ibid., p. 44). Destutt de Tracy montre, à la manière de Beattie, que les modes indicatif, conditionnel, subjonctif, optatif, impératif, interrogatif, et dubitatif, servent tous à exprimer des jugements. La nouveauté ici est l'introduction d'un mode dubitatif, qui est d'ailleurs introduit sur un ton dubitatif: "Je ne crois pas que l'on doive faire un mode particulier de ces tournures de phrases, oserais-je observer? ne pourrait-on essayer? Mais si on le veut, peu importe ( $(i b i d .$, p. 48). Quant au «fond de l'expression », ces phrases veulent dire : «Je doute», «Je ne sais », «Je crois pouvoir », etc. Les modes participe et infinitif ne servent pas à exprimer des jugements; le participe "est un véritable adjectif », et l'infinitif «n'est, pour ainsi dire, pas un mode du verbe; c'est un vrai substantif » (p. 49). Il semble que pour l'auteur, les modes indicatif, conditionnel et subjonctif expriment des modalités d'existence (positive, conditionnelle et subordonnée), et que les autres modes définis servent à former des propositions à propos de nousmêmes : «Je souhaite que... », ou «Je regrette que... » (optatif) ; «Je veux », «Je désire que vous fassiez... ( (impératif) ; «Je demande », «Je désire savoir si...» (interrogatif) ; «Je doute», «Je ne sais» (dubitatif).

\section{Conclusion}

Les linguistes (par exemple Benveniste ${ }^{58}$ et Zaefferer ${ }^{59}$ ) et les philosophes du langage (par exemple, Wittgenstein, Austin, Searle et Vanderveken) affirment qu'on trouvera, dans toutes les

58. E. Benveniste, Problèmes de linguistique générale, Paris, éd. Gallimard, 1966, p. 130.

59. Dietmar ZaEfFerer, "The Semantics of Sentence Mood in Typologically Differing Languages ", in Shiro Hattori (éd.) Proceedings of the XIIt International Congress of Linguists, Tokyo, 1984. 
langues humaines, différents types d'illocution, en particulier des phrases déclaratives, impératives (ou jussives), interrogatives, et exclamatives. Traditionnellement, les philosophes ont surtout fait l'étude des énoncés déclaratifs, seuls porteurs des valeurs de vérité, le mode indicatif étant le mode par excellence de la science et de la connaissance en général. Mais depuis les Investigations philosophiques de Wittgenstein, et les travaux d'Austin et Searle, les énoncés non déclaratifs sont l'objet de très nombreuses études par les philosophes et les linguistes. M. Auroux est dans le vrai lorsqu'il affirme que l'essor des théories de l'énonciation au cours des trente dernières années constitue une véritable "révolution » dans l'histoire des sciences du langage ${ }^{60}$. Mais je crois qu'il voit juste également lorsqu'il écrit, dans un texte portant sur l'histoire de la linguistique : «Jusqu'à ce qu'on s'intéresse à l'énonciation, les théories de Port-Royal étaient plus puissantes que celles dont on disposait. ${ }^{61}$

Si la Grammaire Générale classique avait pour fin de rendre compte « de ce qui est commun à toutes les langues», les grammairiens philosophes ne devaient-ils pas rencontrer ces différents types d'illocution dans toutes les langues qu'ils étudiaient et en rendre compte comme du reste? Dans ce cas, quels traitements ont-ils réservé aux modalités illocutoires? Quelles approches ont été développées, dans le cadre d'une théorie idéationnelle du langage, pour accommoder les « aspects illocutoires de la signification »? C'est pour vérifier cette conjecture et répondre à ces questions que j'ai entrepris cette recherche.

C'est, nous l'avons vu, dans la théorie générale des modes verbaux que les grammairiens philosophes ont débattu ces questions. Nous avons distingué deux approches. L'une, réductionniste, s'apparente aux théories de ceux qui, à la manière de Lewis et Davidson ${ }^{62}$, limitent la sémantique à un traitement des conditions de vérité; dans cette perspective, toutes nos énonciations se

60. À ce propos, S. Auroux, "Actes de pensée et actes linguistiques dans la Grammaire Générale », op. cit.

61. S. Auroux, "L'histoire de la linguistique ", in Langue française, déc. 1980, p. 9.

62. D. Davidson, "Moods and Performances", in A. Margalit (éd.), Meaning and Use, Dordrecht, Reidel, 1976. 
réduisent, après analyse, à l'expression de jugements. L'autre approche, celle des marqueurs d'actes de pensée, se développe, en France, sous l'influence de Port-Royal et Dumarsais, et en GrandeBretagne, de Harris à Gregory. Cette approche distingue nettement le modus (« un mode grammatical » chez Gregory) du dictum (« la matière d'un jugement possible », ou ce qu'il y a de commun dans «Marche! " et «J'ordonne que tu marches»), comme chez Wittgenstein, Austin, Stenius, Searle et Vanderveken. Les modes grammaticaux marquent les forces illocutoires les plus importantes et les plus fréquemment utilisées; ils déterminent si l'usage littéral d'un énoncé doit compter comme affirmation, question, commandement, expression de souhait, etc. Mais le mode ne détermine pas, par exemple, si une affirmation est un témoignage, une prédiction ou un reportage, ou si l'usage d'une phrase impérative exprime une concession, un ordre, un commandement, ou une prière. Les modes déterminent le but illocutoire d'une énonciation littérale, et par le fait même sa direction d'ajustement ${ }^{63}$; mais ils laissent indéterminés certains paramètres illocutoires (le mode d'accomplissement du but illocutoire, les conditions préparatoires, les conditions sur le contenu propositionnel et le degré de puissance des conditions de sincérité). Ce qui n'empêche pas les grammairiens philosophes de discuter à l'occasion de ces autres paramètres, et même d'aborder des questions relatives à la pragmatique des modes. La théorie générale des modes verbaux, particulièrement l'approche des marqueurs d'actes de pensée, constitue un véritable embryon de théorie illocutoire et peut être considérée comme le digne ancêtre des théories actuelles, même si, bien sûr, certains phénomènes énonciatifs lui échappent ${ }^{64}$. Elle vise manifestement la même cible que la théorie des actes de parole : rendre compte,

63. G. Nuchelmans, Judgment and Proposition (1983), urilise à quelques reprises les catégories de la théorie des actes de discours dans son interprétation des philosophes du langage de l'âge classique, notamment celles de force illocutoire et de direction d'ajustement d'une énonciation. D'après lui, la direction d'ajustement est l'un des critères que Dumarsais urilise pour distinguer les propositions des énonciations. J'ajoure qu'il en est de même chez J. Harris (Hermès..., 1751), qui remarque que le mode de demande (qui regroupe l'impératif, le déprécatif et l'interrogatif), contrairement aux modes indicatif, potentiel et subjonctif, exige toujours une réponse !

64. Cf. S. Auroux, "Actes de pensée et actes linguistiques dans la Grammaire Générale ", op. cit., à propos de la « sui-référentialité » qui échappe aux théories idéationnelles du langage. 
d'une manière naturelle et unifiée, des divers types possibles d'illocution. De plus, la théorie des actes de parole reconnaît maintenant (cf. Vanderveken, 1988) l'existence d'actes illocutoires qui peuvent être accomplis en pensée seulement ; les actes illocutoires ne sont pas seulement des unités de communication; ce sont aussi des unités de pensée conceptuelle. La théorie des actes de discours se rapproche par là de la théorie classique des actes de pensée. Dans la direction inverse, l'évolution de la théorie classique des actes de pensée, surtout chez les common sense philosopbers Reid et Gregory, reconnaît finalement l'existence d'opérations sociales de l'esprit, comme le commandement et l'interrogation - qu'on trouve déjà dans la Grammaire de Port-Royal —, ou les requêtes, supplications, serments, avertissements, conseils, exhortations, etc., dont la liste recoupe en bonne partie celle des actes illocutoires.

Pour faire une étude complète de la sémantique des énoncés non déclaratifs dans la Grammaire Générale classique, il faudrait aborder plusieurs autres doctrines grammaticales, examiner les interjections, les pronoms interrogatifs, la grammaire du conjonctif "que », la théorie des propositions, de la conjugaison, etc. Mais je crois avoir montré suffisamment que la théorie générale des modes verbaux constitue vraiment le cœur de cette sémantique idéationnelle des modes d'énoncé. Nos jeunes théories de l'énonciation ont-elle quelque chose à apprendre de cent cinquante ans de Grammaire Générale? Ne peuvent-elles mettre à profit la sagacité, l'érudition, l'imagination théorique, les analyses, les intuitions, les procédés et les exemples des plus illustres grammairiens de l'époque classique? Sait-on jamais...

Département de philosophie

Université du Québec à Trois-Rivières 


\section{BIBLIOGRAPHIE}

AARSLEFF, H. (1967) : The Study of Language in England, 1780-1860, Princeton, Princeton U. Press.

AARSLEFF, H. (1982) : From Locke to Saussure, Minneapolis, U. of Minnesota Press.

ALSTON, W. (1964) : The Philosophy of Language, Englewood Cliffs, PrenticeHall.

ANDRESEN,J.T. (1978) : «François Thurot and the First History of Grammar », in Historiographia linguistica, $\mathrm{V}: 1 / 2$.

ARISTOTE (1966): De l'Interprétation, trad. et notes de J. Tricot, Paris, Vrin.

ARNAULD, A. et LANCELOT, C. (1660): Grammaire générale et raisonnée, éd. critique de $\mathrm{H}$. Brekle, impression en facsimilé de la troisième édition de 1676, Stuttgart-Bad Cannstatt, chez Friedrich Frommann, 1966. 1970.

,et Nicole, P. (1662) : La Logique ou l'art de penser, Paris, Flammarion,

AUGUSTIN (1964) : Les Confessions, Paris, Garnier-Flammarion.

AUROUX, S. (1973) : L'Encyclopédie. «Grammaire » et «Langue » au XVIII siècle, Paris, Mame.

- , (1979) : La Sémiotique des Encyclopédistes, Paris, Payot.

, (1980) : “L'histoire de la linguistique», in Langue française, déc.

, (1985) : «Le temps verbal dans la Grammaire Générale », chap. 1 de Innovation et système, manuscrit.

, (1986) : «Actes de pensée et actes linguistiques dans la Grammaire Générale ", in Histoire. Épistémologie. Langage, VIII : 2.

AUSTIN, J.L. (1962) : How to Do Things with Words, Oxford, Clarendon Press.

BEATTIE, J. (1783): The Theory of Language, Londres, réimpression en facsimilé, Menston, Scolar Press, 1968.

BEAUZÉE, N. (1767): Grammaire générale, ou Exposition raisonnée des éléments nécessaires du Langage pour servir de fondement à l'étude de toutes les langues, 2 vols., Paris, Bardou.

_ , art. "Mode», in l'Encyclopédie, 1765.

BENVENISTE, E. (1966) : Problèmes de linguistique générale, Paris, Gallimard.

BERKELEY, G. (1710): Traité sur les Principes de la connaissance bumaine, trad. et notes de A. Leroy, Paris, Aubier, éd. Montaigne, 1969.

BUFFIER, C. (1709) : Grammaire françoise sur un plan nouveau, Paris. 
BURNETT, J. (Lord Monboddo), (1773-1792): Of the Origin and Progress of Language, Edinburgh, 6 vols. [1773, vol. I ; 1774, vol. II], Menston, Scolar Press, 1967.

BURSILL-HALL, G.L. (1971): Speculative Grammar of the Middle Ages. The Doctrine of partes orationis of the Modistae, La Haye-Paris, éd. Mouton.

CHEVALIER, J.-C. (1968): Histoire de la syntaxe. Naissance de la notion de complément dans la grammaire française (1530-1750), Genève, Droz.

CHOMSKY, N. (1966) : Cartesian Linguistics. A Chapter in the History of Rationalist Thought, New York et Londres, Harper \& Row ; La linguistique cartésienne, suivi de La Nature formelle du langage, trad. de N. Delanoë et D. Sperber, Paris, Seuil, 1969.

CONDILLAC (1947-1951): CEuvres philosophiques, Tome XXXIII du Corpus général des philosophes français, en trois vols., texte établi et présenté par G. Le Roy, Paris, P.U.F.

CORDEMOY, G. de (1666) : Discours physique de la parole, in CEuvres philosophiques, éd. critique de P. Clair et F. Girbal, Paris, P.U.F., 1968.

DAVIDSON, D. (1976) : "Moods and Performances", in A. Margalit (éd.), Meaning and Use, Dordrecht, Reidel, PP. 9-20.

DESCARTES, R. (1637) : Discours de la méthode, Intr. et notes de E. Gilson, Paris, Vrin, 1966. , (1641): Méditations métaphysiques, Paris, P.U.F., 1974.

(1644): Principes de Philosophie, in CEuves philosophiques de Descartes, Tome III, Garnier Frères, Paris, 1973.

DESTUTT DE TRACY, A (1803) : Grammaire, Tome 2 des Élémens d'Idéologie, Paris, Vrin, 1970.

DIDEROT et D'ALEMBERT (1751) : Encyclopédie, ou Dictionnaire raisonnée des sciences, des arts et des métiers; édition conforme à celle de Pellet in quarto, à Berne et Lausanne, chez Les Sociétés Typographiques, 1780.

DOMINICY, M. (1984): La Naissance de la grammaire moderne, Bruxelles, Pierre Mardaga.

DONZÉ, R. (1967) : La Grammaire générale et raisonnée de Port-Royal, Berne, Francke.

DUCHESNEAU, F. (1982) : La Physiologie des Lumières. Empirisme, modèles et théories. La Haye, Martinus Nijhoff.

DUMARSAIS, C.C. (1753) : art. “Construction》 de l'Encyclopédie. , (1769) : Logique et Principes de Grammaire, Paris.

ELSTER, J. (1979) : Ulysses and the Sirens. Cambridge : C.U.P.

FLORA, V. (1980) : "Existe-t-il un mode présomptif en roumain? 》, in Langage et psychomécanique du langage (pour Roch Valin), éd. par A. Joly et W. Hirtle, Lille, Presses de l'U. de Lille. 
GIRARD, G. (1747): Les Véritables Principes de la langue françoise, ou la parole réduite en méchode conformément aux lois de l'usage, en seize discours, Paris.

GREGORY, J. (1790): Theory of the Moods of Verbs, in Transactions of the Royal Society of Edinburgh.

GRICE, H.P. (1975) : « Logic and Conversation », in P. Cole et J.L. Morgan (éd.), Syntax and Semantics, vol. 3, Academic Press.

GRIMSLEY, R. (1971): Sur l'Origine du langage, Genève, Droz.

HARRIS, J. (1751): Hermès, ou Recherches philosophiques sur la grammaire universelle, trad. et Remarques de F. Thurot (1796), éd. par A. Joly avec une Introd, Genève-Paris, Droz, 1972.

HARSANYI, J.C. (1976): Essays on Ethics, Social Bebavior, and Scientific Explanation, Dordrecht, Reidel.

HUME, D. (1748) : Enquête sur l'entendement bumain trad. A. Leroy, Paris, Aubier, Éditions Montaigne, 1947.

HUSSERL, E. (1901): Recherches logiques, Tome second, Paris, P.U.F., 1962.

JOLY, A. et STEFANINI, J. (1977) : La Grammaire générale. Des Modistes aux Idéologues, Lille, Presses U. de Lille.

KASHER, A. (1976) : "Conversational Maxims and Rationality", in Language in Focus, éd. par Kasher, Dordrecht, Reidel.

LAKATOS, I. (1978) : Philosophical Papers, éd. par J. Worrall et G. Currie, Cambridge, C.U.P.

LANCELOT, C (1644) : Nouvelle méthode pour apprendre facilement et en peu de temps la langue latine, Paris.

LECLERC, A. (1988) : “Le problème de la traduction au siècle des Lumières: obstacles pratiques et limites théoriques", in Traduction. Terminologie. Rédaction. Vol. 1, $\mathrm{n}^{\circ} 1$ (1 $^{\mathrm{er}}$ semestre 1988).

LEIBNIZ, G.W. (1765): Nouveaux essais sur l'entendement bumain, Paris, Garnier-Flammarion, 1966.

LEWIS, D. (1972) : «General Semantics», in D. Davidson et G. Harman (éd.), Semantics of Natural Language, Dordrecht, Reidel.

LOCKE, J. (1690) : An Essay Concerning Human Understanding, New York, Dover Publications, Inc., Vol. II, 1959.

MAINE DE BIRAN (1815) : «Note sur les Réflexions de Maupertuis et Turgot au sujet de l'origine des langues", in Sur l'Origine du langage, éd. par R. Grimsley, 1971.

MARTINET, A. (1985) : Syntaxe générale, Paris, Coll. U, Armand Colin.

MAUPERTUIS, P.L. Moreau de (1748) : Réflexions philosophiques surl'origine des langues et la signification des mots, in Varia linguistica, éd. par C. Porset, Bordeaux, éd. Ducros, 1970; aussi dans Grimsley (éd.), 1971. 
MERLEAU-PONTY, M. (1953 et 1960) : "Sur la Phénoménologie du langage ", in Éloge de la Pbilosophie, et autres essais. Paris, Gallimard.

MICHAEL, I. (1970): English Grammatical Categories and the Tradition to 1800, Cambridge, C.U.P.

NUCHELMANS, G. (1973): Theories of Propositions. Ancient and Medieval Conceptions of the Bearers of Truth and Falsety, Amsterdam, NorthHolland

(1983) : Judgment and Proposition. From Descartes to Kant, Amsterdam, North-Holland.

PADLEY, G.A. (1985): Grammatical Theories in Western Europe: 1500-1700, Cambridge, C.U.P.

PARRET, H. (éd.), (1976): History of Linguistic Thought and Contemporary Linguistics, Berlin, de Gruyter.

PARIENTE, J.-C. (1985) : L'Analyse du langage à Port-Royal, Six études logicogrammaticales, Paris, Éd. de Minuit.

PORSET, C. (éd.), (1970) : Varia linguistica, Bordeaux, Ducros.

QUINTILLIEN (1954): Institution oratoire, Tome 1, trad. de H. Bornecque, Paris, Garnier Frères.

RAWLS, J. (1971): A Theory of Justice, Cambridge (Mass.), The Belknap Press of Harvard.

REID, T. (1785): Essays on the Intellectual Powers of Man, Cambridge (Mass.) et Londres, Presses du M.I.T., 1969.

RESTAUT, P. (1730): Principaux généraux et raisonnés de la grammaire françoise, Paris.

RICHARDS, D. (1971): A Theory of Reasons for Action, Oxford, O.U.P.

RICKEN, U. (1978) : Grammaire et philosopbie au siècle des Lumières : Controverse sur l'ordre naturel et la clarté du français, Villeneuve-d'Ascq, Université de Lille III.

ROUSSEAU, J.J. (1754): Discours sur l'origine de l'inégalité parmi les bommes (avec $D u$ Contrat social et le Discours sur les sciences et les arts), Paris, Union Générale d'Édition, 1963.

SAHLIN, G. (1928) : César Cbesneau Du Marsais et son rôle dans l'évalution de la grammaire générale, Paris, P.U.F.

SAUSSURE, F. de (1915): Cours de linguistique générale, Paris, Payot, 1922.

SCHREYER, R. (1978) : “Condillac, Mandeville, and the Origin of Language», in Historiographia linguistica, V: $1 / 2$.

SEARLE, J. (1979) : Expression and Meaning. Studies in the Theory of Speech Acts, Cambridge, C.U.P.

, et VANDERVEKEN, D. (1985) : Foundations of Illocutionary Logic, Cambridge, C.U.P. 
SGARD,J. (éd.), (1982): Condillac et les problèmes du langage, Genève et Paris, Slatkine.

SIMON, H. (1957) : Models of Man, New York, John Wiley \& Sons, Inc.

STENIUS, E. (1967): "Moods and Language Game", in Synthese, 17, p. $254-274$.

THUROT, F. (1796) : "Remarques " à sa Traduction de Hermès, ou Recherches philosophiques sur la grammaire universelle, de J. Harris, éd. par A. Joly, Genève, Droz, 1972.

(1796): Tableau des progrès de la science grammaticale (Discours préliminaire à Hermès), éd. par A. Joly, Bordeaux, éd. Ducros, 1970.

TURGOT, A.-R. (1750) : «Remarques critiques sur les Réflexions philosophiques de Maupertuis sur l'origine des langues et la signification des mots», in Varia linguistica, éd. par C. Porset.

VANDERVEKEN, D. (1988) : Les Actes de discours. Essai de philosophie du langage sur la signification des énonciations, à paraître chez Pierre Mardaga.

WATKINS, J. (1970) : «Imperfect Rationality », in R. Borger et F. Cioffi (éd.), Explanation in the Behavioural Sciences, Cambridge, C.U.P.

WITTGENSTEIN, L. (1953) : Philosophical Investigations, Oxford, Blackwell.

ZAEFFERER, D. et GREWENDORF, G. (1984a) : "Theorien der Satzmodi ", art. du manuel (Handbuch) Semantik, éd. par D. Wunderlich et A, von Stechow, manuscrit.

ZAEFFERER, D. (1984b) : "The Semantics of Sentence Mood in Typologically Differing Languages ", in Shiro Hattori (éd.), Proceedings of the XIII International Congress of Linguists, Tokyo, 1984.

ZUBER, R. (1983) : Non-Declarative Sentences, Amsterdam, John Benjamins Publishing Compagny. 